\title{
Sozialstaatliche Umverteilung und ihre Akzeptanz im internationalen Vergleich: Eine Mehrebenenanalyse Redistribution by the Social Welfare State and Public Support for It -
What Explains Country Differences?
}

\author{
Ursula Dallinger* \\ Universität Trier, Abteilung Soziologie, 54286 Trier, Germany. \\ E-Mail: dallinge@uni-trier.de
}

\begin{abstract}
Zusammenfassung: Der Artikel fragt, wie das international variierende Niveau der Akzeptanz von Ungleichheit und staatlicher Einkommensumverteilung in fortgeschrittenen Industriegesellschaften zu erklären ist. Er greift zunächst die in der vergleichenden Forschung zum Wohlfahrtsstaat vertretene Regimetheorie auf, für die der Einfluss von sozialpolitischer Kultur und der von ,cleavages" zentral ist. Da beides weder empirisch bestätigt ist, noch andere Erklärungen für Länderunterschiede berücksichtigt wurden, bezieht der vorliegende Beitrag auch Ansätze der politischen Ökonomie ein. Diese betrachten die Umverteilungspräferenzen als geprägt von der jeweiligen Ungleichheit der Einkommensverteilung (median voter-Hypothese), vom wirtschaftlichen Wohlstand sowie den ,varieties of capitalism'. Erklärungen, die auf der Ebene individueller Merkmale ansetzen, werden theoretisch und empirisch mitgeführt. Da der Einfluss von Länder- und Individualmerkmalen geprüft wird, wird eine Mehrebenenanalyse mit Daten des ISSP 1999 verwendet. Den empirischen Ergebnissen zufolge ist eine Polarisierung der ökonomischen und der Regimeansätze unangemessen. Die Bewertung von Ungleichheit und Umverteilung ist teils durch sozialpolitische Ideen vermittelt, die objektive Ungleichheit überformen, teils ist die Akzeptanz von Umverteilung als Reaktion der Bürger auf länderspezifische Bedingungen zu interpretieren. Besonders wirtschaftlicher Wohlstand mindert die Umverteilungswünsche.
\end{abstract}

Summary: This article seeks to explain international variation in public support of income redistribution by the social welfare state to reduce inequalities. On the one hand, it discusses the welfare regime approach, which explains differences according to variation in political culture and in cleavages in countries. However, previous comparative studies have not been able to confirm a regime approach empirically; furthermore, they neglect other explanations and do not control for other national features relevant to redistribution demands of their citizens. Consequently, this contribution includes approaches from political economics, that understand redistributional preferences as rational reactions of actors to conditions such as the degree of inequality (median voter-hypothesis), economic wealth, and the "varieties of capitalism." Explanations based on individual characteristics are only briefly discussed. Hypotheses about national differences are tested by controlling for individual characteristics based on data from ISSP 1999 and additional data about country characteristics such as GINI, GDP, regimeclusters, etc. Multilevel regression is used because country and individual level data are analysed. According to the results the two approaches cannot be contrasted: neither political economy nor regime approach alone offers an adequate explanation of the data. The impact of the economic factors is mediated by culture and vice versa: Ideas are embedded in economic conditions.

\section{Einleitung}

Die Umverteilung von Einkommen ist eine für den modernen Wohlfahrtsstaat konstitutive Funktion (Kaufmann 2003). Die auf Märkten entstandene, primäre Einkommensverteilung wird durch Sozialbeiträge, durch direkte und indirekte Steuern wie auch durch Sozialtransfers mehr oder weniger stark angeglichen. Dies geschieht nicht nur ideeller Ziele

* Ich danke den anonymen Gutachtern für die hilfreiche Kritik und die Anregungen. Weiter bestehende Mängel des Aufsatzes gehen zulasten der Autorin. wie Egalität wegen, sondern auch zur Vermeidung von Armut oder zur Stärkung des Konsums. Staatliche Umverteilung von den ökonomisch Stärkeren zu den ökonomisch Schwächeren ist jedoch umstritten. Weil sie der ökonomischen Entwicklung schade und Leistungsanreize nehme, wird ihre Legitimität bezweifelt. Außerdem verlangt Umverteilung Opfer von ,den Gewinnern in der großen Lotterie des Marktes, die den Abstand zwischen ihnen und den Verlierern verringern.' (Streeck 2000: 136). Der vorliegende Beitrag untersucht daher, ob und unter welchen Bedingungen ein Mehr an Gleichheit durch Umverteilung von den Bürgern unterstützt wird. Wegen des Drucks auf den Wohlfahrtsstaat 
im verstärkten globalen Wettbewerb und wegen der oft beschriebenen sozialen und kulturellen Pluralisierung kann man bezweifeln, ob es überhaupt noch soziale Strukturen und Wertüberzeugungen gibt, auf deren Grundlage ein Eingreifen des Sozialstaats in den Markt zur Korrektur von Ungleichheit akzeptiert wird (Streeck 2001: 144). ,Public support ${ }^{\circ}$ ist aber eine wichtige Determinante der Spielräume sozialpolitischen Handelns (Brooks/Manza 2006).

Nun weiß man aus der international vergleichenden Forschung zu Einstellungen zum Wohlfahrtsstaat, dass die Unterstützung für staatliche Einkommensumverteilung zugunsten reduzierter Ungleichheit deutlich zwischen verschiedenen Ländergruppen variiert. Vor diesem Hintergrund fragt der Beitrag, wie man das divergierende Niveau der Akzeptanz staatlicher Einkommensumverteilung in fortgeschrittenen Industriegesellschaften erklären kann. Denn die Ergebnisse zu Niveauunterschieden zwischen den verschiedenen Ländern legen nahe, dass nicht nur individuelle Interessen oder Werthaltungen die Zustimmung zu Umverteilung beeinflussen, sondern auch die Gegebenheiten der Länder. ${ }^{1}$ Welche Eigenschaften eines Landes aber genau zu einer unterschiedlichen Beurteilung von Ungleichheit sowie zu unterschiedlicher Akzeptanz sozialstaatlicher Umverteilung führen, ist ungenügend erforscht. Bisher wurde die zwischen Ländern variierende Akzeptanz von Umverteilung vor allem auf der Grundlage der Regimetheorie von Esping-Andersen erklärt, die Varianzen in der Zustimmung zu redistributiver Politik durch die Bürger auf die jeweils in den Regimetypen dominierenden Ideen oder auf Ideologien über Ungleichheit und staatliche Einkommensredistribution zurückführt (vgl. Svallfors 1997, Arts/Gelissen 2001, Mau 2004). Eine andere Variante des Regimeansatzes in der Forschung zu public opinions erklärt die Länderunterschiede bezüglich der sozialpolitischen Präferenzen durch vom Regime geprägte Interessengruppen. ${ }^{2}$ Da das unterschiedliche Niveau der Zustimmung zu Umverteilung aber allein dem Einfluss von Regimen zugeschrieben wird, übersieht man, dass Länderunterschiede ebenso gut aufgrund anderer

\footnotetext{
${ }^{1}$ Auch in Bezug auf andere sozialpolitische Programme oder Prinzipien sozialer Gerechtigkeit, die die Gestaltung von Sozialpolitik beeinflussen, wies die bisherige Forschung typische Varianzen zwischen den Ländern im Umfang des, public support' nach.

2 Siehe Blekesaune/Quadagno 2003, Meier Jaeger 2006a. Die beiden Varianten des regimetheoretischen Ansatzes in der Einstellungsforschung werden in Kap. 3.1 genauer erläutert.
}

Merkmale des Landes entstehen, die bislang unkontrolliert geblieben sind. Der vorliegende Aufsatz will das Spektrum der Erklärungsansätze für länderspezifische Umverteilungspräferenzen der Bürger erweitern und bezieht zusätzlich Ansätze der politischen Ökonomie ein, die das unterschiedliche Niveau der Zustimmung zu Umverteilung in den einzelnen Ländern als aggregiertes Resultat rationaler Reaktionen der Bürger auf ökonomische und institutionelle Rahmenbedingungen betrachten. Danach prägten makro-ökonomische Bedingungen wie die jeweilige Ungleichheit der Einkommensverteilung und das Wohlfahrtsniveau eines Landes die Präferenzen für die weitere Umverteilung von Einkommen. Vor diesem Hintergrund untersucht der Beitrag empirisch, ob Umverteilungspräferenzen eher von den kulturellen Leitideen der Regime geprägt werden oder ob diese eher als rationale, die eigenen Vorteile maximierende Reaktionen von Akteuren im Rahmen von länderspezifischen MakroFaktoren zu deuten sind. Die Hypothese lautet, dass sich beide Erklärungsmuster nicht wechselseitig ausschließen, sondern komplementär sind, da die Einstellung zu sozialstaatlicher Umverteilung sowohl in kulturelle als auch in ökonomische Kontexte eingebettet ist.

Neben den Erklärungen für das zwischen Ländern variierende Toleranzniveau gegenüber Einkommensumverteilung sind selbstverständlich auch jene Forschungsprogramme wichtig, die auf individuelle Faktoren wie die von der sozialen Position des Einzelnen beeinflussten Interessen oder die Werthaltungen der Bürger in Bezug auf Egalität oder Solidarität abstellen. Sie bleiben in diesem Beitrag im Hintergrund, werden aber konzeptionell und empirisch mitgeführt. Da die Unterstützung von Umverteilung sowohl mit individuellen als auch mit Ländermerkmalen erklärt wird, wird das Verfahren der Mehrebenenanalyse verwendet. Mit diesem lässt sich auch die Relevanz der individuellen Merkmale im Vergleich zu den Ländermerkmalen zeigen. Der empirischen Analyse liegen Daten aus der repräsentativen Befragung zu Social Inequality III von 1999 zugrunde, die im Rahmen des International Social Survey Programme (ISSP) durchgeführt wurde. ${ }^{3}$ In dieser wurde u. a. die Wahrnehmung und Bewertung von Einkommensunterschieden wie auch die Zustimmung zu staatlicher Umverteilung zur Reduzierung der Unterschiede zwischen hohen und niedrigen Einkommen erfragt.

\footnotetext{
${ }^{3}$ Die Daten sind beim Zentralarchiv Köln unter der Nummer 3430 archiviert.
} 
Man könnte dem Gegenstand des Beitrags gegenüber grundsätzlich kritisch fragen, ob Wohlfahrtsstaaten tatsächlich umverteilen. Denn sie organisieren zum größten Teil Sozialversicherungen, die gegen individuelle Risiken beim Ausfall des eigenen Einkommens (bei Alter, Krankheit, Arbeitslosigkeit) schützen sollen und primär nur eine intertemporale Umverteilung der Einkommen, aber kaum eine interpersonelle Umverteilung praktizieren. Die Spielräume für soziale Umverteilung sind in der Tat begrenzt. Gleichwohl verteilen Wohlfahrtsstaaten sowohl durch Sozialversicherungen als auch durch Steuern nachweislich um und gleichen Einkommensdifferenzen an, wenn auch im internationalen Vergleich betrachtet in sehr unterschiedlichem Maße (Atkinson et al. 1995, Birkel 2005, Kenworthy/ Pontusson 2005). Außerdem kann man argumentieren, dass die Unterstützung von Umverteilung durch die Bürger ein eigenständiger Gegenstand ist, selbst wenn deren tatsächlicher Umfang gering ist.

,Public support` von sozialstaatlicher Umverteilung bietet einen Zugang zur solidarischen Dimension des Wohlfahrtsstaats. Dieser wird oft insgesamt als ,organisierte Solidarität ${ }^{`}$ bezeichnet (Lessenich 1999), oder die Solidaritätspotenziale werden abgelesen an der Zustimmung zu Mehrausgaben für die Alterssicherung (Gelissen 2001). Beides ist jedoch ungenau. Denn statt dem Wohlfahrtsstaat generell das Attribut „solidarisch“ zuzuweisen, differenziert die Unterscheidung in einen soziale Sicherheit leistenden Anteil und in einen umverteilenden Anteil des Sozialstaats angemessener zwischen dem Mix an individuellen Sicherungsinteressen und lebenszeitlicher Umverteilung sowie den durchaus auch umverteilenden Aspekten staatlicher Sozialpolitik, die die Solidarität der Bürger beanspruchen (Offe 1990). Solidarische Anteile sind meines Erachtens an einem primär auf die eigene Einkommenssicherheit abstellenden Programm (wie die Alterssicherung) nicht gut zu lokalisieren. ${ }^{4}$ Statt dessen bietet die von einzelnen sozialpolitischen Programmen abstrahierende Frage nach der Zustimmung zu sozialstaatlicher Umverteilung (wie sie ISSP, ESS oder Eurobarometer verwenden) einen direkteren $\mathrm{Zu}$ griff auf die Solidaritätspotenziale der Bürger. Legt man eine Definition des Solidaritätsbegriffs zugrunde, die vom Begriffsursprung im römischen Schuldrecht ausgeht (Metz 1998, Bayertz 1998), dann

\footnotetext{
${ }^{4}$ Auch Sozialversicherungen enthalten mit der Umverteilung etwa von den Gesunden zu den Kranken oder von Personen mit einem niedrigen zu jenen mit einem hohen Arbeitslosigkeitsrisiko solidarische Elemente (Ullrich 2000, Hinrichs 1997).
}

wird die Verbindung zwischen Solidarität und Umverteilung deutlich, denn danach bedeutet Solidarität die Verpflichtung einer Gruppe, für ,zahlungsunfähige' Mitglieder mit aufzukommen (in solidus). ${ }^{5}$ Von hier ausgehend lässt sich der Bogen zu der sozialpolitisch relevanten Frage schlagen, ob und wie viel Verantwortung die Gruppe (der Beitrags- und Steuerzahler) für ,Zahlungsunfähige“ übernehmen soll, was eben impliziert, das eigene Einkommen mit diesen zu teilen (Bowles/Gintis 2000). Die ,Mithaftung des Kollektivs' setzt voraus, dass die Gruppe einen Teil ihrer Ressourcen zugunsten schwächerer Mitglieder verteilen kann und will. Umverteilung bedeutet, den ,Gewinnern' der ökonomischen Entwicklung, Opfer im Namen sozialer Solidarität ${ }^{`}$ abzuverlangen (Streeck 2001: 151). Sie verlangt einen Beitrag zum Gemeinwohl, den der Staat erzwingen können muss und der gleichwohl als legitim betrachtet wird (van Oorschot 2000). ${ }^{6}$

Im Folgenden werden zunächst Ansätze und Ergebnisse der Forschung zur Akzeptanz staatlicher Umverteilung und Solidarität rekonstruiert (2). Dabei geht der Beitrag sowohl auf die in der politischen Soziologie verankerten regimetheoretischen Erklärungen (2.1) als auch auf die in der politischen Ökonomie verankerten Ansätze ein (2.2). Danach wird gezeigt, mit welchen individuellen Merkmalen die inter-individuellen Variationen bei der Akzeptanz von Umverteilung erklärt werden (2.3). Es folgt eine Darstellung der methodischen Vorgehensweise, der Datengrundlage, der Indikatoren sowie der ausgewählten Länder (3). Die empirischen Analysen ergeben, dass sich die Annahmen von kulturellen Einflüssen einerseits und von einer rationalen Ausbildung von Umverteilungspräferenzen andererseits nicht gegeneinander ausspielen lassen. Unterstützung für Umverteilung wird durch sozialpolitische Ideen, die in bestimmten Regimetypen institutionalisiert sind, und durch rationale Reaktionen des ,median voters' geprägt (4). Die Schlussfolgerungen (5) fassen die Ergebnisse zusammen.

\footnotetext{
${ }^{5}$ Dem etymologischen Ursprung des Solidaritätsbegriffs im römischen Recht zufolge beschrieb der Begriff, in soli$d u s^{\text {' }}$ eine schuldrechtliche Haftungspflicht der Gruppe für einzelne zahlungsunfähige Mitschuldner.

6 "Societal solidarity increases the more the strong' support the ,weak “" (Arts/Gelissen 2001: 285).
} 


\section{Die Akzeptanz von Umverteilung - Variationen zwischen Ländern und Individuen}

\subsection{Der regimetheoretische Ansatz}

Das Regimekonzept wurde ursprünglich von Esping-Andersen (1996) [1990] zur Erklärung der zwischen den westlichen Industrieländern variierenden Sozialpolitikmuster entwickelt. Die Forschung $\mathrm{zu}$ den Einstellungen gegenüber dem Wohlfahrtsstaat adaptierte die regimetheoretische Perspektive zur Erklärung auch der empirisch oft nachgewiesenen Länderunterschiede bei den sozialpolitischen Präferenzen. Es gebe ,typisch' liberale, konservative und sozialdemokratische Einstellungsmuster gegenüber Sozialpolitik. Zum einen wird dies mit den in Regimes institutionalisierten sozialpolitischen Ideen und Werten und speziell auch mit Vorstellungen von angemessener und gerechter Umverteilung begründet, die letztlich die Einstellungen in der Bevölkerung prägten. ${ }^{7}$ Zum anderen bildeten sich in den Regimes spezifische cleavages, die ein Interesse an wohlfahrtsstaatlicher Umverteilung entwickelten. Beide Varianten sind näher zu erläutern.

Nach Esping-Andersen sind Regime Bündel institutioneller Praktiken und Programme, die sich in der Art und Weise unterscheiden, in der sie soziale Sicherung gestalten, Ungleichheit beeinflussen und umverteilen. Weil sich die regimespezifischen Sozialpolitiken wie auch die steuerliche Umverteilung auf Ungleichheit auswirken, bezeichnet Esping-Andersen (1996: 36) den Wohlfahrtsstaat als ein System der Stratifizierung, denn der Wohlfahrtsstaat verändert die primäre, auf Märkten entstandene Ungleichheit und erzeugt selbst wieder spezifische Muster der Ungleichheit im Zuge der sekundären Einkommensverteilung. Regime variieren zudem in dem Grad, in dem wohlfahrtsstaatliche Transfers eine Alternative zur Existenzsicherung allein über den Arbeitsmarkt bieten (,de-commodification'). Die verschiedenen Regime entwickelten sich historisch in westlichen Industriegesellschaften unter dem Einfluss der jeweils dominanten politisch-gesellschaftlichen Kräfte und ihrer Koalitionen. Wohlfahrtsregime entstanden aufgrund der in einzelnen Ländern unterschiedlichen Machtressourcen der Interessenorganisationen verschiedener gesellschaftli-

\footnotetext{
${ }^{7}$ Diese Einführung kann der Debatte um die Stimmigkeit der Unterscheidung von Wohlfahrtsregimen nicht gerecht werden. Der Schwerpunkt muss in diesem Beitrag darauf liegen, wie das Regimemodell in der Einstellungsforschung rezipiert wurde; vgl. dazu Kaufmann 2003: $22 \mathrm{f}$.
}

cher Gruppen. Die dominanten Interessengruppen haben Esping-Andersen zufolge jeweils solche wohlfahrtsstaatlichen Arrangements etabliert, die für ihre Klientel vorteilhaft waren und ihren sozialpolitischen Ideologien entsprachen. Im Ergebnis korrelieren in Regimen deshalb ein bestimmter $\mathrm{Zu}$ schnitt wohlfahrtsstaatlicher Institutionen, die Koalitionen sozialer Gruppen sowie eine spezifische ideologische Tradition (Esping-Andersen 1996: 138). So betonen liberale Regime die private Verantwortung für die eigene Einkommenssicherung und intervenieren nur wenig in die durch marktförmige Prozesse entstandene Verteilung. Konservative Regime sind demgegenüber geprägt durch die katholische Soziallehre und die Christdemokratie als dominante politische Kraft (Becker/Kersbergen 1986), weswegen sie eine stärkere Verantwortung für die soziale Sicherheit der Einzelnen übernehmen und stärker auf Umverteilung setzen als liberale Regime. Da jedoch die Sicherung des jeweiligen Status angestrebt wird, hat die Umverteilung Grenzen. In sozialdemokratischen Regimen schließlich ist nach Esping-Andersen durch die starke Stellung der Arbeiterparteien die Umverteilung besonders ausgeprägt. Weil sozialdemokratische Kräfte auf Koalitionsbildung angewiesen waren, seien die sozialen Anrechte umfassend und auch privilegierteren Schichten zugänglich. Diese Regimetypologie wurde um südeuropäisch-mediterrane und post-sozialistische Regime erweitert (Lessenich/Ostner 1998, Andreß/Heien 2001). Die Bürger des letztgenannten Typs sollten hohe Erwartungen an einen für die Wohlfahrt seiner Bürger verantwortlichen Staat haben und einen ausgeprägten Egalitarismus vertreten (Kluegel/Myano 1995, Braun 1998, Delhey 1998). Mediterrane Regime hingegen sind schwer einzuordnen; sie ähneln dem konservativen Typus, ergänzt durch eine familialistische Komponente, die aber im Kontext von Umverteilung nicht relevant ist.

Esping-Andersen betrachtete Regime aus der Sicht eines konflikttheoretischen Ansatzes (Korpi 1983) als Ergebnis der Machtressourcen der gesellschaftlichen Interessengruppen. Inzwischen betonen aber Studien zum Vergleich von Sozialstaaten (oder von Sozialpolitikmustern in einzelnen Feldern), dass Regime sowohl durch die in der Phase ihrer Entstehung und Entwicklung dominierenden Interessengruppen geprägt werden als auch durch spezifische sozialpolitische Ideen und Ideologien (Taylor-Gooby 2001). Es wird der Einfluss organisierter Interessen und ebenso der Einfluss sozialpolitischer Ideen gezeigt, wobei die letzte Lesart des Regimeansatzes im Zuge des Aufkommens neo-institutionalistischer 
Theorien in den letzten Jahren Auftrieb erhielt. ${ }^{8}$ Auch die am Regimeansatz ausgerichteten PublicOpinion-Forschung fragt zum einen, ob man in verschiedenen Regimen typische cleavages findet (a), und zum anderen, ob Ideen der Bürger über Gerechtigkeit und Umverteilung zwischen verschiedenen Regimen variieren (b).

a) Aufgrund der Anreize, die von wohlfahrtsstaatlicher Verteilung ausgehen, entwickelten sich in Regimen cleavage structures mit unterschiedlichen Interessen an Umverteilung. So lasse das sozialdemokratische Regime mit einem ausgebauten öffentlichen Dienstleistungssektor, der v.a. Frauen Beschäftigungschancen gibt, eine überproportionale Unterstützung von Umverteilung durch Frauen erwarten. Konservative Regime hingegen begünstigten die soziale Sicherung der Beschäftigten, so dass sich die Umverteilungspräferenzen der Insider und der Outsider des Arbeitsmarktes gegenüberstehen. Für liberale Regime sei zu erwarten, dass primär die untere Klasse ein Interesse an umverteilender sozialstaatlicher Intervention hat. Empirisch fand man allerdings keine typischen cleavages in den einzelnen Regimen. Differenzen nach Geschlecht, Klasse sowie den In- und Outsidern des Arbeitsmarktes strukturierten vielmehr in allen Ländern gleichermaßen die Unterstützung für staatliche Umverteilung: „When it comes to differences between groups, it is rather the similarities between regime types than the differences that are noteworthy" (Svallfors 1997: 295, Taylor-Gooby 2001). Neuere Studien versuchen, mit einem ausgefeilteren methodischen Vorgehen dennoch, cleavages' nachzuweisen (Linos/West 2003, Meier Jaeger 2006b). In diesem Beitrag brauchen uns die Ergebnisse nicht im Einzelnen zu beschäftigen, da die Unterschiede in den , cleavages' nach wie vor ungesichert sind. ${ }^{9}$

b) In der zweiten Interpretation und Weiterentwicklung der Regimetheorie wird betont, dass in wohlfahrtsstaatlichen Regimen sozialpolitische Ideen

\footnotetext{
${ }^{8}$ Zur Verankerung des Wohlfahrtsstaates in kulturellen und insbesondere religiösen Leitideen siehe Kaufmann 1997:141ff., 2003: 35ff., Rieger/Leibfried 2004, im Hinblick auf Reziprozitätsprinzipien siehe Lessenich 1999, Mau 2002.

${ }^{9}$ Linos und West veränderten in ihrer Replikation der ursprünglichen Studie von Svallfors (1997) die Behandlung fehlender Werte und die Operationalisierung der Klassen. Nur partiell fanden sie die erwarteten ,Cleavagestrukturen' in Einstellungen zu Einkommensverteilung. Meier Jaeger (2006b) analysiert ,Cleavagestrukturen ' auf der Grundlage eines kanadischen Paneldatensatzes. Mit einem einzelnen Land lässt sich aber nicht prüfen, ob Regime sich durch je typische ,cleavages' auszeichnen.
}

institutionalisiert sind, die die Einstellungen der Bürger gegenüber Umverteilung und Verteilungsgerechtigkeit beeinflussen (Svallfors 1997, Gelissen 2001, Mau 2004). Der Einfluss der Regime auf die Ideen und Urteile der Bürger über den Wohlfahrtsstaat wird als formativer Effekt bezeichnet, der von einer den wohlfahrtsstaatlichen Institutionen inhärenten „moralischen Logik“ ausgehe. Regime „tend to promote different ways of valuing market distribution and the redistributive responsibilities of government" (Svallfors 1997: 286) und beeinflussen so die Vorstellungen der Bürger von Solidarität und Gerechtigkeit langfristig (Arts/Gelissen 2001, Gelissen 2002). Formelle Institutionen des Wohlfahrtsstaats bestehen demzufolge nicht nur aus sozialpolitischen Maßnahmen, vielmehr verkörpern sie auch spezifische Ideen darüber, was ein Wohlfahrtsstaat tun soll, wie viel Ungleichheit akzeptabel ist und was gerecht ist. ${ }^{10}$ Denn sozialstaatliche Institutionen seien unter dem Einfluss sozialpolitischer Ideologien entstanden, die sich langfristig in nationalen historical legacies verfestigten, die man als Komplex aus spezifischen Ideen und wohlfahrtsstaatlichen Programmen verstehen kann. Die institutionellen Arrangements eines Regimes gestalten nach dieser Auffassung die Beziehungen zwischen Bürgern nach spezifischen Ideen, und sie beeinflussen so die Einstellungen (Mau 1997: 44, 2004). Nach dem Regimeansatz lautet eine erste Hypothese:

$\mathrm{H}$ 1: Wenn es einen Einfluss von Regimen auf die Präferenzen gibt, dann müsste einmal das Niveau der Zustimmung zu Umverteilung in den zu einem Regime gehörenden Ländern konsistent sein. Weiter sollte folgende Hierarchie sichtbar werden: Bürger liberaler Länder sollten Umverteilung am wenigsten tolerieren, während konservative und mediterrane Regime ein mittleres Niveau an Umverteilungspräferenzen aufweisen sollten. Für sozialdemokratische Länder ist im Vergleich zu den zuvor genannten Ländergruppen eine höhere Akzeptanz staatlicher Umverteilung zu erwarten; in post-sozialistischen Regimen dürfte wegen der noch verankerten sozialistischen Ideologie die Unterstützung für Umverteilung am höchsten sein.

Empirische Ergebnisse bestätigen den regimetheoretischen Ansatz zur Erklärung der Umverteilungspräferenzen der Bürger jedoch nur teilweise. Entgegen der Erwartung, dass Bürger sozialdemokratischer Regime die stärkste ,Neigung' zur Umverteilung haben sollten, sind gerade sie es, die re-

${ }^{10}$ Die unterschiedlichen Leitideen der Gestaltung des Verhältnisses zwischen Markt, Staat und Familie bezeichnet Taylor-Gooby (2001) als ,welfare values'. 
distributive Maßnahmen relativ wenig unterstützen (Svallfors 1997: 289). Dagegen fand sich bei Bürgern mediterraner Staaten wie Spanien oder Portugal ein hohes Niveau an Unterstützung für Umverteilung (Gelissen 2002). Dies ist insofern plausibel, als es in diesen weniger ausgebauten, ,unreifen" Wohlfahrtsstaaten noch Spielräume für ein Mehr an Umverteilung gibt. Auch steht die Unterstützung für den Abbau der Einkommensungleichheit in unreifen Wohlfahrtsstaaten mit hoher Ungleichheit in einem ganz anderen Kontext als etwa in Schweden, wo Ungleichheit stark reduziert ist. In einer auf die Länder Deutschland, Norwegen und die Vereinigten Staaten beschränkten Analyse ließ sich gleichwohl die erwartete regimespezifische Toleranz für Redistribution nachweisen (Andreß/Heien 2001). Bei einer so geringen Zahl an Fällen sind Verallgemeinerungen aber schwierig.

Inzwischen gibt es verschiedene Kritiken an den Versuchen, von Länderdifferenzen unkontrolliert auf einen Regimeeinfluss zu folgern (Lippl 2003). Die Gleichsetzung von Land und Regime in der regimevergleichenden Forschung ist für Meier Jaeger (2006a) der Grund dafür, dass man die erwartete Beziehung zwischen Umverteilungspräferenzen und Regimen bislang nicht fand. Er setzt deswegen nicht Länder als Proxy-Variable für Regime ein, sondern Indikatoren für den spezifischen Politikstil der Regime. Das sind der Umfang öffentlicher Sozialausgaben, das Gewicht sozialer Dienstleistungen im Verhältnis zu Geldleistungen, die Höhe der Geldund Dienstleistungen für Familien und die Lohnersatzrate der Arbeitslosenunterstützung. Aber auch mit diesen Indikatoren als erklärende Variablen und mit einer Mehrebenenanalyse fanden Meier Jaeger wie auch andere Studien (Svallfors 1997, Gelissen 2001, Arts/Gelissen 2001) keine Korrespondenz zwischen Regimen und den Einstellungen zu Redistribution. Indikatoren, die nach den Hypothesen typisch für sozialdemokratische Regime sind und positiv mit hohen Redistributionspräferenzen korreliert sein sollten, weisen eine negative Korrelation auf. So haben etwa hohe Sozialausgaben für Sachleistungen und ein hohes Lohnersatzniveau, beides kennzeichnet sozialdemokratische Regime, einen negativen Einfluss auf die Unterstützung für Umverteilung. Nur zum Teil fand Meier Jaeger die erwarteten Zusammenhänge, die für hohe Gleichheitspräferenzen beim ausgebauten sozialdemokratischen Staat sprechen: Je höher der Anteil der Sozialausgaben am Bruttosozialprodukt ist, desto mehr unterstützen Bürger Redistribution. Das Resultat, dass im Kontext sozialdemokratischer Politikmerkmale die Unterstützung für Redistribution eher schrumpft, spricht aber eindeutig gegen eine monokausale Prägung durch Regime. Erst mithilfe der unten diskutierten Annahmen zu Sättigungseffekten wird die negative Korrelation plausibel. ${ }^{11}$

Die referierten empirischen Resultate zeigen, dass sich länderspezifische Einstellungen der Bürger keineswegs vollständig aus einer Prägung durch historical legacies ergeben. Die Hypothese, Umverteilungspräferenzen seien geformt von den in Regimen institutionalisierten sozialpolitischen Ideen, geht ausschließlich vom Modell des homo sociologicus aus. Befunde wie die negative Korrelation zwischen hohem Lohnersatzniveau und der Unterstützung von Umverteilung weisen vielmehr darauf hin, dass dieses um den homo economicus ergänzt werden muss.

\subsection{Umverteilungsbedarf als Reaktion auf ökonomische Bedingungen der Länder}

Die zwischen Ländern variierende Unterstützung für Umverteilung kommt keineswegs allein aufgrund unterschiedlicher kultureller Wahrnehmungsfilter zustande, denn Länder setzen auch in anderer Hinsicht Rahmenbedingungen, die für das jeweils präferierte Niveau an Umverteilung relevant sind wie das Ausmaß der Ungleichheit bei der Einkommensverteilung oder der wirtschaftlichen Entwicklung. Makro-ökonomische Variablen werden in der Politischen Ökonomie zur Erklärung sozialpolitischer Präferenzen herangezogen, aber auch in empirischen Studien eher ad hoc aufgegriffen. Gemäß den Ansätzen der Politischen Ökonomie reagieren Bürger rational auf die in ihrer Umwelt vorfindbaren Bedingungen und bilden solche Einstellungen bzw. Präferenzen gegenüber (Un-)Gleichheit und Umverteilung aus, die ihrem individuellen Nutzen entsprechen.

Eine solche rationale Reaktion wird mit der Sättigungshypothese beschrieben, der zufolge in Ländern, die in hohem Maße umverteilen und ,reife', ausgebaute Wohlfahrtsstaaten repräsentieren (wie Schweden), eine gewisse, Sättigung' des Bedarfes an Einkommensangleichung erreicht sei. Weitere Umverteilung werde weniger gewünscht, weil die bereits geringe Ungleichheit der Einkommensverteilung kaum noch Umverteilungsmotive mobilisiere

\footnotetext{
${ }^{11}$ Meier Jaeger berichtet „perplexing results“ (2006a: 167) auch zu weiteren Indikatoren. So korrelieren großzügige Leistungen für Familien keineswegs mit der Unterstützung für Redistribution, sondern umgekehrt nimmt diese $a b$.
} 
und der Grenznutzen weiterer Umverteilung abnehme. Zudem wirke sich die hohe Belastung der Steuer- und Beitragszahler negativ auf Umverteilungsforderungen aus. Dem entsprechen empirische Ergebnisse, wonach in Ländern mit einer geringen Ungleichverteilung der Einkommen (gemessen mit dem Gini-Koeffizient oder -index ${ }^{12}$ ) Umverteilung weniger unterstützt wird (Roller 1995, Lippl 2003). Auch die so genannte, median voter'-Hypothese von Meltzer und Richard (1981) basiert auf dem Einfluss des nationalen Ungleichheitsniveaus auf individuelle Umverteilungspräferenzen, die dann in aggregierter Form die Länderunterschiede ergeben. Ihren Namen bezieht diese These aus der Überlegung, dass Personen mit Medianeinkommen bei demokratischem Mehrheitswahlrecht ihr Interesse an Umverteilung durchsetzen können. Ist die Einkommensungleichheit groß, dann liegen die Bezieher von Medianeinkommen mit Abstand unter dem Durchschnittseinkommen, und die Mehrzahl der Wähler kann von Umverteilung, die üblicherweise durch progressive Steuern finanziert wird, eindeutig profitieren und wählt Umverteilung. Wenn aber durch Umverteilung das Median- an das Durchschnittseinkommen heranrücke, verringere sich das Interesse an Umverteilung, da bei zunehmender Angleichung der Einkommensabstände das Risiko wachse, durch Steuern selbst Umverteilung finanzieren zu müssen. Aus der, median voter -Hypothese ergibt sich ein dynamisches Modell der langfristigen Entwicklung der gesellschaftlichen Forderungen nach mehr Gleichheit und Umverteilung: Wird die Einkommensungleichheit zu groß, fordern Bürger mit unterdurchschnittlichem Einkommen über Wahlen einen Ausgleich, dem die politischen Parteien wegen ihres Interesses am Gewählt-Werden nachkommen; die Ungleichheit sinkt dann wieder. ${ }^{13} \mathrm{Ab}$ einem bestimmten Punkt jedoch lassen Umverteilungsforderungen nach. Statisch ausgedrückt besteht gemäß der median voter-Hypothese eine positive Beziehung zwischen dem Ungleichheitsniveau eines Landes

\footnotetext{
12 Der Gini Index ist eine Zahl zwischen 0 und 1. Ein niedriger Gini Index zeigt eine gleichere Einkommensverteilung an, eine hohe Zahl dagegen stärker ungleich verteilte Einkommen.

${ }^{13}$ Eine eingehendere Diskussion der ,median voter'-Hypothese findet man bei Kenworthy/MacCall (2007). Die ,problem response hypothesis 'von Stjerno und Johannessen (2004) weist in die gleiche Richtung wie die Annahme über die Präferenzen des Durchschnittswählers, denn sie postuliert einen Zusammenhang zwischen einem sozialen Problem und einer entsprechenden ,Antwort'. Bürger reagierten auf Probleme wie hohe Arbeitslosigkeit oder hohe Armutsquoten mit Zustimmung zu sozialstaatlichen Ausgaben für entsprechende Maßnahmen.
}

(Gini) und der Zustimmung zu Umverteilung, so dass die Hypothese gilt:

H 2: Je höher der Gini-Index eines Land ist, desto höher ist der Wunsch nach Umverteilung (und umgekehrt).

Viele Sozialforscher haben die Hypothese, dass Bürger bei hoher Ungleichheit ein hohes Maß an staatlicher Umverteilung fordern, aber mit Hinweis auf die USA, in denen es trotz hoher Ungleichheit wenig Unterstützung für Umverteilung gibt, bezweifelt (Bowles/Gintis 2000, Kangas 2003, Kenworthy/ Pontusson 2005). Hohe Ungleichheit zieht dort gerade keine umfassenden Umverteilungsforderungen nach sich. Manche Autoren erklären dies mit einer ,redistributive ethics' (Bowles/Gintis 2000) oder mit den zwischen Ländern differierenden Normen der Verteilungsgerechtigkeit (Lübker 2007). Aber auch andere als kulturelle Erklärungsmöglichkeiten sind plausibel. So können Bürger - obwohl die Ungleichheit hoch ist - dennoch nicht die Notwendigkeit eines staatlichen Gegensteuerns in Betracht ziehen, sondern sehen in Arbeitsmarktbeteiligung die Lösung. Die hohe Ungleichheit in den USA kann sich auch wegen der starken ethnischen Spaltungen nicht in Umverteilungsforderungen umsetzen, was nach Alesina et al. (2001) die den Erwartungen widersprechende Situation dort erklärt. Die geringen Umverteilungsforderungen bei gleichzeitig hoher Ungleichheit in den USA erfordern eine spezifische Erklärung. Diese besondere Konstellation darf aber nicht dazu führen, das Modell der (Wähler-)Forderungen nach Umverteilung als einer rationalen Reaktion auf Ungleichheit völlig zu verwerfen. Andere Studien (Finseraas 2006) und auch die später gezeigten eigenen Ergebnisse bestätigen durchaus, dass der Grad der Ungleichheit die Umverteilungspräferenzen partiell beeinflusst.

Eine weitere Erklärung dafür, dass die Umverteilungspräferenzen der Bürger sich nicht mechanisch aus Ungleichheit ableiten, bietet die asset theory of social policy preferences von Iversen und Soskice (2001). Dieser im Rahmen der varieties of capitalism entwickelte Ansatz erklärt internationale Unterschiede in den Präferenzen für sozialstaatliche Ausgaben für soziale Sicherung (nicht für Umverteilung!) vor dem Hintergrund verschiedener product market regimes. Diese repräsentieren unterschiedliche Typen der Wirtschaft mit spezifischer Ausrichtung der Produktion, der Arbeitsmarktorganisation und mit einem jeweils dominierenden Beschäftigtentypus. In den auf einfache Produkte und Dienstleistungen ausgerichteten liberalen Marktwirtschaften (LME) überwögen Beschäftigte mit generellen Qualifikationen, deren Investition in Ausbildung 
gering sei. Weil generelle Qualifikationen leicht auf andere Arbeitsplätze transferierbar seien, berge ein Arbeitsplatzverlust nur geringe Risiken, weshalb diese Gruppe sozialstaatliche Absicherung wenig nachfrage. Dagegen überwögen in koordinierten Marktwirtschaften (CME), die technologisch anspruchsvolle Produkte herstellen, firmen- und industriespezifisch spezialisierte Beschäftigte mit höheren Qualifikationen. Aber gerade diese Gruppe der Beschäftigten befürworte höhere staatliche Sozialausgaben, da sie ein Interesse an einem hohen Lohnersatzniveau habe; denn bei Arbeitsplatzverlust müsse dieser Beschäftigtentyp längere Sucharbeitslosigkeit auf einem unflexiblen Arbeitsmarkt überbrücken (Hall/Soskice 2001: 51). Mit diesem Modell kann die asset-theory zwar das geringe $\mathrm{Ni}$ veau an Umverteilungspräferenzen in liberalen Ländern erklären, das dem median voter-Ansatz Schwierigkeiten bereitet. Die Umverteilungspräferenzen in koordinierten Marktökonomien kann sie aber weniger gut erschließen. So müssten etwa in Deutschland besser Qualifizierte Umverteilung unterstützen, was aber dem Befund widerspricht, dass gerade Unqualifizierte einen umverteilenden Wohlfahrtsstaat befürworten. Anscheinend kann man die asset theory, die ja die Zustimmung zu Ausgaben für soziale Sicherung erklärt, nur partiell auf Präferenzen für Umverteilung übertragen. Eine weitere Einschränkung ist die Konzentration dieser Theorie auf Beschäftigte und ihre Interessen an sozialer Absicherung bei Arbeitslosigkeit. Erwartungen in Hinblick auf Umverteilungsvorteile entwickeln aber auch die Nicht-Erwerbstätigen.

Dass Ungleichheit die Einstellungen zu Ungleichheit und Umverteilung nicht direkt beeinflusst, zeigen auch Arbeiten zum Einfluss der institutionellen Gestaltung des Sozialstaats (Rothstein 1998, Korpi/ Palme 2001, Moene/Wallerstein 2001). Ob faktische Ungleichheit $\mathrm{zu}$ Umverteilungsforderungen führt, hängt danach vom Zuschnitt staatlicher Transferleistungen ab. Bei einer primär auf die Bedürftigsten ausgerichteten Sozialpolitik entwickle sich selbst bei hoher Ungleichheit nur wenig Unterstützung für Sozialausgaben, da ,benefit targeting ${ }^{\circ}$ den Kreis der Benefiziare und Befürworter einschränkt. ${ }^{14}$ Der breiten Masse der Bürger zukommende Sozialausgaben (und die dafür nötigen Steu-

${ }^{14}$ Korpi und Palme (2001) verknüpften die Streuung der sozialstaatlichen Geld- und Dienstleistungen auf die breite Mittelschicht nicht nur mit hoher Akzeptanz sozialstaatlicher Ausgaben, sondern zeigten auch eine größere Wirkung in Hinblick auf die tatsächlichen Umverteilungseffekte. Da nicht die auf die Benachteiligten gerichteten Systeme viel Gleichheit schaffen, sondern die Leistungen ern und Beiträge) würden dagegen eher akzeptiert, da sie der eigenen (Sozial-)Versicherung dienen. Ob Sozialpolitik eher ,targeted' ist oder aber dem Sozialversicherungstyp entspricht, kommt in der Höhe der Sozialausgaben (als Anteil am BIP) zum Ausdruck. Bei hohen Sozialausgaben hätten wir demnach mehr Akzeptanz von Umverteilung zu erwarten. Obgleich es sinnvoll wäre, den Einfluss von Sozialausgaben zu prüfen, verzichtet die vorliegende Analyse wegen der geringen Länderzahl darauf.

$\mathrm{Zu}$ den Bedingungen, die für die Politische Ökonomie die Umverteilungspräferenzen beeinflussen, gehört außerdem der wirtschaftliche Wohlstand eines Landes. Die ökonomische Prosperität eines Landes schränke den Bedarf der Bürger für Redistribution ein, weil bei steigendem Einkommensniveau staatliche Umverteilung seltener als notwendig wahrgenommen werde (Haller et al. 1990: $35 \mathrm{ff}$.$) . Denn Wohlstand schwäche die mit Armut$ und ungleichen Lebensverhältnissen einhergehenden sozialen Probleme ab und lasse diese weniger gravierend erscheinen. Ein hohes Bruttoinlandsprodukt (BIP) führe zu einer Sättigung im Sinne eines Nachlassens des Drucks zu weiterer Umverteilung. Entsprechende Studien sprechen für die Annahme, dass Bürger in Ländern mit hohem BIP pro Kopf Redistribution weniger unterstützen. Da diese Beobachtung anhand einer begrenzten Auswahl an Ländern und nicht unter Kontrolle weiterer Ländervariablen gemacht wurde, kann dieser Beitrag den Zusammenhang aufgrund der größeren Länderzahl breiter prüfen. Die dritte Hypothese lautete daher:

H 3: Je höher das Wohlstandsniveau (BIP pro Kopf) eines Landes ist, desto geringer ist die Zustimmung zu Umverteilung, da eine Sättigung des Bedarfs an Umverteilung eintritt.

Wir sahen, dass Länderunterschiede bei den politischen Einstellungen gegenüber staatlicher Umverteilung entweder mit den jeweils dominierenden sozialpolitischen Ideen über (Un-)Gleichheit oder staatliche Verantwortung erklärt werden oder aber als Ergebnis des Einflusses unterschiedlicher Rahmenbedingungen - dazu gehören Einkommensungleichheit, Wohlstandsniveau und unterschiedliche Produktionssysteme - auf rationale Akteure interpretiert werden. Ich gehe hingegen davon aus, das sich beide Effekte - der Einfluss von sozialpolitischen Ideen und die Anreize makro-ökonomischer Bedingungen - überlagern.

breit streuenden, sprachen sie von einem ,Umverteilungsparadox'. 


\subsection{Variationen zwischen Individuen: Interessen und Wertorientierungen}

Homo oeconomicus und homo sociologicus tauchen aber nicht nur in Erklärungen von Länderunterschieden auf, sondern auch in Studien, die die Akzeptanz von Ungleichheit und Umverteilung anhand individueller Merkmale erklären (TaylorGooby 1983, 1985, Andress/Heien 2001; Linos/ West 2003, Blekesaune/Quadagno 2003, Meier Jaeger 2006). Für die Politische Ökonomie sind es die Interessen Einzelner, die das Ausmaß der Unterstützung der sozialstaatlichen Redistribution beeinflussen. Ein Interesse an Umverteilung haben zum einen Personen mit direkter Abhängigkeit von staatlichen Transfers und zum anderen Personen, die aufgrund ihres geringen Einkommens oder hohen Arbeitslosigkeitsrisikos Vorteile durch Umverteilung erwarten. Empirisch bestätigt ist, dass es eher Arbeiter und eher Personen mit geringer Qualifikation sowie niedrigem Einkommen oder Leistungsempfänger sind, die Redistribution befürworten und demnach von Umverteilung zu profitieren hoffen (Wilensky 1973, Bean/Papadakis 1998, Svallfors 1997, Corneo/Gruner 2002). Rehm (2005) untersuchte angelehnt an das Modell der asset-spezifischen Präferenzen von Iversen-Soskice, ob Präferenzen für staatliche Umverteilung von berufsspezifischen Arbeitsmarktrisiken abhängen. Beschäftigte mit spezifischen beruflichen Qualifikationen, die hohen Arbeitsmarktrisiken ausgesetzt sind, fragten Umverteilung stärker nach als Beschäftigte mit leicht auf andere Arbeitsplätze transferierbaren generellen skills (auch Iversen/Soskice 2001). Er fand in der Tat einen Zusammenhang zwischen berufsspezifischen Arbeitslosigkeitsrisiken und Umverteilungswünschen. Im Kontext dieses Aufsatzes soll aber das Interesse an Umverteilung und erwarteten Transfers nicht nur für Beschäftigte, sondern allgemeiner geprüft werden, da Abhängigkeit von Transfers aus verschiedenen Gründen wie etwa Arbeitslosigkeit, geringer Qualifikation und geringem Einkommen entstehen kann. Die vierte Hypothese lautet vor diesem Hintergrund:

H 4: Personen bzw. soziale Gruppen, die Vorteile von Umverteilung erwarten oder besonders auf diese angewiesen sind, haben höhere Umverteilungspräferenzen.

Das heißt im Einzelnen: a) Ein niedriges Bildungsniveau, das schlechtere berufliche Chancen und höhere Arbeitsmarktrisiken nach sich zieht, b) ein niedriges Einkommen und c) eine niedrige Position in der sozialen Hierarchie führen zu einem größeren Interesse an sozialpolitischer Umverteilung.
Neben dem homo oeconomicus, der wohlfahrtsstaatliche Umverteilung nach den individuellen Vorteilen beurteilt, spielt in der Forschung zur Akzeptanz von Redistribution der homo sociologicus eine wichtige Rolle. Demnach beurteilten Bürger den ,richtigen' Umfang an Einkommensangleichung auf der Basis von kulturellen Ideen (Roller 1995, Andress/Heien 2001, van Oorschot 2001). Das Argument für Kultur lautet, dass Umverteilungspräferenzen kein unvermittelter Reflex auf die objektive Lage (Arbeitslosigkeit, Einkommen), sondern stets von Wertvorstellungen bzw. politischen Ideologien vermittelt seien. Objektive Gegebenheiten würden vor dem Hintergrund von Ideen über Gerechtigkeit und Solidaritätsnormen bewertet (Offe 1987, Rothstein 1998, Mau 2002, 2004; Blekesaune/Quadagno 2003). Auch die neo-institutionalistische Perspektive unterstreicht Werte, da für sie formale Institutionen des Wohlfahrtsstaats eine moralische oder kulturelle Basis haben. Corneo und Gruner (2002) begreifen Werte als social incentives, die den Wunsch erzeugten, in Übereinstimmung mit öffentlich anerkannten Normen zu handeln. Empirische Ergebnisse bestätigen, dass politische Ideologien bzw. Ideen bezüglich ,gerechter' Verteilung oder bezüglich der Gründe für sozialen Aufstieg die Unterstützung von Umverteilung beeinflussen. Naheliegenderweise befürworten Anhänger linker politischer Parteien eher eine staatliche Umverteilung (Meier Jaeger 2006a). Politische Ideen werden allerdings mit Parteipräferenzen nur grob erfasst. Die neuere Forschung versuchte daher, einen direkteren Zugang zu verteilungsrelevanten Ideen zu gewinnen. Dabei erwiesen sich Egalitarismus (Blekesaune/Quadagno 2003) oder Ideen über die Voraussetzungen des sozialen Aufstiegs (Linos/ West 2003) als einflussreich. In Beiträgen aus der Gerechtigkeitsforschung wird die Beurteilung staatlicher Umverteilung auf von den Bürgern vertretene Gerechtigkeitsprinzipien zurückgeführt. Maßgeblich für die Bewertung der Einkommensverteilung sind demzufolge drei basale Prinzipien der Verteilungsgerechtigkeit equality, equity und need. Personen mit egalitaristischer Grundhaltung stimmen eher dem staatlichen Eingreifen in die Einkommensstrukturen $\mathrm{zu}$, während Verfechter von equity Umverteilung wenig akzeptabel finden, da für sie Einkommen nach individuellen Leistungen im Beruf verteilt sein soll (Kluegel/Miyano 1995). Geprüft wird als fünfte Hypothese:

H 5: Unterstützung für Redistribution wird beeinflusst von spezifischen Gerechtigkeitsideen. Wird der sozialen Ungleichheit eine positive Funktion für den Wohlstand aller zugebilligt, dann sinkt die $\mathrm{Zu}$ stimmung zu Umverteilung. 


\section{Operationalisierung und Daten}

Die Unterstützung für einen umverteilenden Wohlfahrtsstaat wird in diesem Beitrag mit ausgewählten Items aus dem International Social Survey Programme III (ISSP) des Jahres 1999 analysiert. Die abhängige Variable, die Umverteilungsbereitschaft, wird aus zwei Items gebildet. In ihnen sollen Befragte anhand einer fünfstufigen Skala ihre $\mathrm{Zu}$ stimmung oder Ablehnung gegenüber den beiden folgenden Statements bestimmen: „Die Einkommensunterschiede in [Land] sind zu groß.“ „Es ist die Aufgabe des Staates, die Einkommensunterschiede zwischen den Leuten mit hohem und solchen mit niedrigem Einkommen zu verringern.“ Das erste Item erhebt bereits eine Wertung der wahrgenommenen Ungleichheit und tendiert daher deutlich in die Richtung des zweiten Items, das Befragte zu einem Rating auffordert, ob der Staat Einkommensungleichheit reduzieren soll. Man kann eine hohe Konkordanz im Antwortverhalten bei beiden Items annehmen. Diejenigen, die Ungleichheit nicht als zu groß bezeichnen, werden kaum Umverteilung durch den Staat bejahen, und umgekehrt. Daher ist es durchaus legitim, einen Summenindex aus beiden Items $\mathrm{zu}$ bilden. (Dieser schwankt zwischen dem Wert 2 und 10.) Allenfalls kann es eine Diskrepanz zwischen der Bewertung der Ungleichheit und der Bewältigung durch staatliche Umverteilung geben, da man nicht den Staat, sondern die individuelle Arbeitsmarktpartizipation als Ansatzpunkt einer Lösung sieht. Empirisch ist das aber kaum der Fall. Sowohl der inhaltliche $\mathrm{Zu}$ sammenhang beider Items als auch die erwähnte Einschränkung lassen sich statistisch belegen. Beide Items des Index korrelieren hoch signifikant miteinander (.52) und gehören zu einem Faktor. Cronbachs Alpha hat einen Wert von .701 und weist damit den Summenindex als reliabel aus. ${ }^{15}$ Nur in den USA und Kanada wird der Zusammenhang zwischen beiden Einzelitems schwächer, denn der durchschnittliche Skalenwert für das Umverteilungsitem liegt unter dem zur Bewertung von Ungleichheit (siehe Tab. A1 im Anhang), weil offenbar in diesen beiden Ländern die Bewertung der Ungleichheit nicht in dem hohen Maße an Unterstützung staatlicher Umverteilung gekoppelt ist, wie in den anderen Ländern. Dennoch dürfte die Verwendung eines Summenindex die Ergebnisse nicht verzerren. Denn eine Regression mit der Ein-

${ }^{15}$ Variablentransformationen sind dokumentiert in einem download auf der Homepage www.uni-trier.de/index. php?id=15833 zelvariable zur Unterstützung staatlicher Umverteilung allein ergab keine grundsätzlich anderen Resultate.

Die Mittelwerte der abhängigen Variable, dem Index zu Umverteilung, unterscheiden sich deutlich in einzelnen Ländern (siehe Tab. A1 im Anhang). Deshalb und wegen der hierarchischen Datenstruktur wird in diesem Aufsatz eine Multilevel-Regression verwendet. Multilevel- oder Mehrebenenverfah$\operatorname{ren}^{16}$ werden der Tatsache gerecht, dass die Daten der Befragten in einzelne Länder gruppiert sind (,nested $\left.{ }^{6}\right)$, was bei einer OLS-Regression Probleme bereiten würde. Wegen der Mischung von Daten auf zwei Ebenen würden die Standardfehler der Regression zu klein ausfallen, da die Stichprobengröße bei allen Ländervariablen (auch Kontextvariablen genannt) tatsächlich sehr viel kleiner ist als die Zahl der befragten Individuen. Durch die Multilevelanalyse werden die Standardfehler zuverlässiger. Die geringere Zahl der Ländermerkmale würde außerdem dazu führen, dass die Signifikanz der Kontextvariablen überschätzt würde. Wegen der gruppierten Daten wären die Residuen nicht unkorreliert, was eine Grundannahme der Regression verletzt. Außerdem kann man den Beitrag der erklärenden Variablen auf den verschiedenen Ebenen zur Erklärung der Akzeptanz von Umverteilung getrennt schätzen. Verwendet wird ein Random Intercept und Random Slope Modell, bei dem die Regressionskonstanten der Länder und auch die Steigungen bestimmter Regressionsgeraden zufällig schwanken (Rabe-Hesketh/Skrondal 2005, Hans 2006). Es werden also zum einen fixe Effekte für die erklärenden Variablen geschätzt. Zum anderen gehe ich von Zufallseffekten bei der Regressionskonstanten ${ }^{17}$ und den Steigungen der beiden unabhängigen Variablen Geschlecht und Einkommen aus. Mit der Variable ,Geschlecht' im ,random part ${ }^{\star}$ der Multilevel-Regression wird angenommen, dass das $\mathrm{Ge}$ schlecht die Umverteilungspräferenzen in verschiedenen Ländern unterschiedlich stark beeinflussen dürfte. Mit der Variable ,Familieneinkommen' im zufälligen Teil der Regressionsgleichung kann man die Annahme modellieren, dass das Einkommen nicht in allen Ländern gleich stark die Unterstützung für Einkommensumverteilung mindert. Weiter wurden im RS-Modell mit Hilfe von zwei Interaktionsvariablen Cross-Level-Effekte analysiert. Allgemein wird mit Cross-Level-Effekten modelliert,

\footnotetext{
16 Zur Mehrebenenanalyse vgl. Engel 1998.

17 D. h. die länderspezifischen Werte der abhängigen Variable ,Umverteilungspräferenzen“ variieren zufällig um einen durchschnittlichen Wert.
} 
dass Kontextvariablen einen Teil der Varianz der Steigungskoeffizienten der individuellen Variablen erklären. Im Kontext dieses Beitrags ist die Annahme sinnvoll, dass der Einfluss des Familieneinkommens auf die Umverteilungspräferenzen abhängig vom jeweiligen BIP und Gini-Koeffizienten des Landes schwankt. Mit steigendem BIP dürfte sich der negative Effekt des Einkommens verstärken, da Wohlstand Umverteilung weniger notwendig erscheinen lässt. Hingegen sollte mit steigender Ungleichheit der Einkommensverteilung der Effekt des Einkommens abgeschwächt sein.

Die in die Analyse eingehenden Länder bilden eine erweiterte Regimetypologie ab. Schweden und Norwegen sind als sozialdemokratische Regime einbezogen, Frankreich, Österreich und Westdeutschland als konservative, Großbritannien, die Vereinigten Staaten, Kanada, Australien, Neuseeland und Japan als liberale Regime. Über Esping-Andersens klassische Typologie hinaus werden - basierend auf der Literatur zu deren Ergänzungsbedürftigkeit (z. B. Lessenich/Ostner 1998) - mit Spanien, Zypern und Portugal mediterrane Regime, und mit Polen, Russland, Slowenien, Slowakei, Bulgarien, Lettland, Ungarn, Ostdeutschland und der Tschechischen Republik post-sozialistische Regime ${ }^{18}$ aufgenommen. Ostdeutsche werden wegen der fortbestehenden ideellen Unterschiede über Wohlfahrtsstaat und Ungleichheit dem postsozialistischen Typus zugeordnet. Damit sind insgesamt 23 Länder und 27.437 Fälle Grundlage der empirischen Analyse. In der zunächst deskriptiven Beschreibung der zwischen ihnen variierenden Umverteilungspräferenzen und ihren Strukturen werden die Länder noch nicht zusammengefasst, um innerhalb eines Regimetyps mögliche Variationen sichtbar zu machen. In die multivariate Mehrebenenanalyse gehen die Länder jedoch gebündelt zu Regimen und als DummyVariablen ein.

Weitere Ländermerkmale, die als unabhängige Variable der zweiten Ebene berücksichtigt werden, sind erstens die Ungleichheit der Einkommensverteilung, gemessen mit dem Gini-Index. Informationen zum Gini-Index stammen soweit wie möglich aus der Luxembourg Income Study (LIS), die aber nicht alle in dieser Studie aufgenommenen Länder

${ }^{18}$ Deren Regimestatus wird bezweifelt, da sie eher vorübergehende Formen der Wohlfahrtsstaatlichkeit seien. Dieser Einwand ist aber für die hier behandelte Fragestellung nicht gravierend. Auch ist die Zuordnung einzelner Länder zu bestimmten Regimen nicht eindeutig. M.E. würden aber diese uneindeutigen Länder das Ergebnis der Analyse nicht grundsätzlich ändern. abdeckt. Ergänzende Daten zu weiteren Ländern kommen aus EUROSTAT und für Japan werden Daten der OECD zurate gezogen (Förster/d'Ercole 2005). Stets handelt es sich um den Gini-Index für das bedarfsgewichtete Haushaltsnettoeinkommen. Da der Gini nicht immer genau für das Jahr der Befragung (1999) verfügbar ist, wurden die zeitlich nächstliegenden Werte verwendet. Zweitens geht als Indikator für die Wirtschaftskraft eines Landes das BIP pro Kopf 1999 (in Dollar, Purchasing Power Parity = ppp; siehe HDR 2003) in die Regression ein.

Unabhängige Variablen auf der individuellen Ebene sind Indikatoren zum einen für Interessen, zum anderen für Werteinstellungen. Die Interessen werden erschlossen mit der Höhe des Haushaltseinkommens in Dezilen (1-10), der subjektiven sozialen Position (von $1=$ hoch bis $10=$ niedrig) und der Bildung in Jahren. Da das schulische Bildungsniveau eng mit beruflichen Chancen zusammenhängt, kann Letzteres auch als eine Proxy-Variable für die mit dem Bildungsmangel steigenden Arbeitsmarktrisiken und Risiken des Einkommensverlusts gelesen werden. Die Dummy-Variable zu Geschlecht soll den bekannten Zusammenhang kontrollieren, dass Frauen sozialstaatliche Ausgaben und Umverteilung stärker unterstützen, und zusätzlich im Rahmen des RSModells zwischen den Ländern variierende Effekte prüfen. Denn nach dem Regime-Ansatz fördern sozialdemokratische Regime, dass die besonders durch die Verteilungsstrukturen begünstigten Frauen entsprechende Umverteilungspräferenzen entwickeln. ${ }^{19}$ Individuelle Wertorientierungen werden mit einem Item erfasst, das die Funktion von Ungleichheit anspricht und formuliert, dass die Ungleichheit der Einkommen dem Wohlstand aller nutze (,Ungleichheit fördert Wohlstand').

\section{Die Akzeptanz von Umverteilung in ökonomischen und ideellen Kontexten}

Ein erster Schritt analysiert Unterschiede in der Akzeptanz staatlicher Umverteilung zunächst noch für die einzelnen Länder, bevor diese im zweiten Schritt zusammengefasst zu Regimen in eine multivariate

${ }^{19}$ Ursprünglich berücksichtige das Modell auch die Arbeitsmarktpartizipation als Dummy (nicht Erwerbstätige, Arbeitslose und Rentner vs. Erwerbstätige), weil angenommen worden war, dass Transfers abhängige Ungleichheit kritisch bewerten und Umverteilung fordern. Da dieser Effekt aber weit weg von der Signifikanzgrenze war, wurde er zugunsten eines sparsamen Modells weggelassen. 


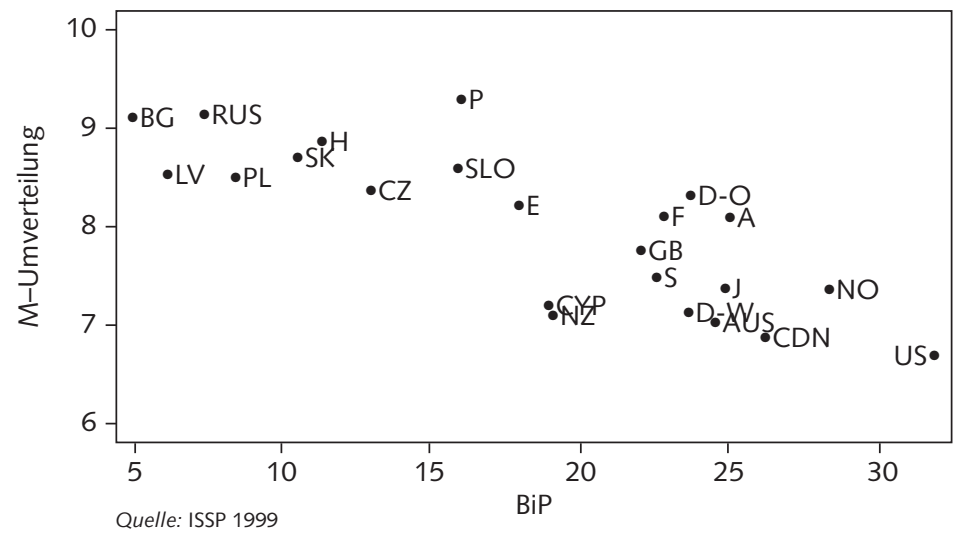

Abb.1 Zustimmung zu Umverteilung nach BIP.
Analyse mit dem gepoolten Datensatz eingehen. Dadurch lassen sich Diskrepanzen innerhalb einzelner Regime erkennen, die später nicht mehr sichtbar sind, jedoch einen wichtigen Hintergrund für die Interpretation der Ergebnisse der multivariaten Analyse darstellen.

Anhand von Tabelle A1 im Anhang erkennt man deutliche Unterschiede zwischen den Ländern in der Bewertung von Ungleichheit und der $\mathrm{Zu}$ stimmung der Bevölkerung zu Umverteilung. Die Mittelwerte des Umverteilungsindexes variieren zwischen den einzelnen Ländern deutlich. Sie schwanken zwischen einem Minimalwert von 6,69 für die Vereinigten Staaten und einem Maximalwert von 9,27 für Portugal. Die Mittelwerte der postsozialistischen und mediterranen Länder stehen an vorderster Stelle, die der liberalen Länder liegen am Ende, die sozialdemokratischen und konservativen Länder nehmen eine mittlere Position ein. Die Rangordnung entspricht weitgehend den Erwartungen, mit Ausnahme der Position der sozialdemokratischen Länder, wo wenig (weiterer) Umverteilungsbedarf besteht. Die nach der Regime-Hypothese zu erwartende Konsistenz der zum gleichen Regime gehörenden Länder im Niveau der Unterstützung von Umverteilung ist aber kaum zu finden. Nur die postsozialistischen Länder weisen ein einheitliches Niveau auf, während andere Regime wenig homogen sind. Die dem liberalen Regime zugerechneten Länder (USA, Kanada, Australien, Neuseeland, GB) sind nur eingeschränkt homogen, weil Großbritannien ,ausreißt ${ }^{\star}$ und deutlich höhere Umverteilungspräferenzen aufweist als seine liberalen Partner. Auch die Gruppe konservativer Länder ist in Bezug auf das Niveau der Kritik an Ungleichheit und daraus folgenden Umverteilungsforderungen inkonsistent: Westdeutsche liegen mit deutlichem Abstand unter Franzosen und Österreichern. Das
Unterstützungsniveau für Umverteilung unter Westdeutschen ähnelt eher dem in liberalen Ländern, das der Ostdeutschen dem in postsozialistischen Ländern. Man findet also nur teilweise homogene „Welten der Umverteilungspräferenzen“. Die postsozialistischen und auch die liberalen Länder (Großbritannien ausgenommen) jedoch bilden deutliche Cluster. ${ }^{20}$

Die Annahme, dass das Wohlstandsniveau eines Landes die Unterstützung für Umverteilung beeinflusst, erhält mehr empirische Bestätigung (siehe Abb.1). Denn im Großen und Ganzen gibt es eine klare Tendenz, dass die Umverteilungswünsche bei hohem wirtschaftlichen Reichtum sinken und eine ,Sättigung' einsetzt. Allerdings bestehen innerhalb der ,reichen“ Länder deutliche Unterschiede in den Umverteilungspräferenzen. So befürworten Franzosen, Österreicher und Norweger im Durchschnitt trotz wirtschaftlichen Wohlstands Umverteilung stärker als die Bevölkerungen anderer wohlhabender Länder (etwa Japan, Deutschland, Kanada). Da ähnliche ökonomische Rahmenbedingungen Umverteilungswünsche also nicht determinieren, dürften sozialpolitische Ideen eine wichtige Rolle dabei spielen, wie objektive Bedingungen - hier wirtschaftlicher Wohlstand - von Bürgern ,interpretiert' werden.

${ }^{20}$ Da meine Auswertung die Asset-Theorie der Sozialpolitikpräferenzen aus der Varieties of capitalism-Forschung nicht ins Zentrum stellt, verschiebe ich den folgenden Befund in eine Fußnote: Dass das Niveau der Umverteilungswünsche in den meisten liberalen Marktökonomien (Kanada, Neuseeland, Australien) unter dem koordinierter Marktökonomien (Norwegen, Schweden, Österreich und Frankreich) liegt, bestätigt den Einfluss von product-market-regimes auf Ungleichheitsbewertung und Umverteilungspräferenzen teilweise. Da Westdeutschland nicht ins Bild passt, erklären auch Produktionsregime nicht alle Aspekte. 


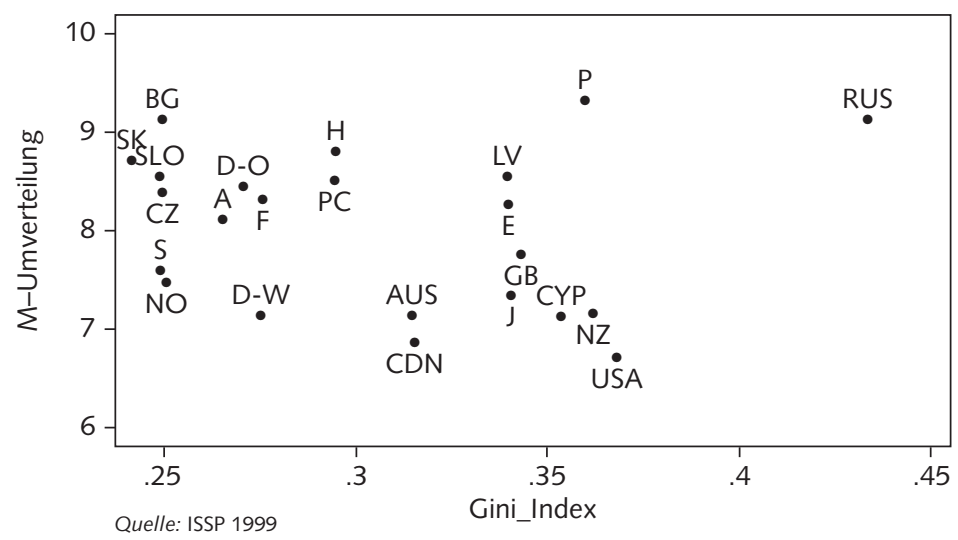

Abb. 2 Zustimmung zu Umverteilung nach Gini-Index.

Außerdem sollte es nach den konzeptionellen Überlegungen eine positive Beziehung zwischen dem Grad an Ungleichheit und der Befürwortung staatlicher Umverteilung geben, also bei starken Diskrepanzen in der Einkommensverteilung und einem hohen Gini-Index auch hohe Umverteilungspräferenzen und umgekehrt. Betrachtet man Abbildung 2, dann trifft diese positive Beziehung für die im unteren linken Bereich platzierten Länder zu (Westdeutschland, Norwegen, Schweden). Ein niedriger Wert beim Gini-Index und geringe Umverteilungspräferenzen konvergieren, wie das die ,median voter-Hypothese annimmt. Eine analoge positive Beziehung zwischen hohem Gini und umfassenden Umverteilungsforderungen weist Abbildung 2 aber nur für Russland und Portugal aus. Die Lage der anderen Länder stellt jedoch in Frage, ob Ungleichheit ein Interesse der ,median voter ${ }^{6}$ an Umverteilung erzeugt. Alle Länder, die nicht in der Nähe einer gedachten Diagonale von links unten nach rechts oben liegen, sperren sich dieser Erklärung. Das sind einerseits die USA und andere liberale Länder im Bereich rechts unten, wo trotz Ungleichheit die Bevölkerung wenig Umverteilung fordert, und andererseits postsozialistische und konservative Länder links oben, wo selbst bei geringer Ungleichheit weiterhin das Unterstützungsniveau für Umverteilung hoch ist. Die bivariate Verteilung bestätigt Hypothese 2 vorläufig nur teilweise und zwar für die spezifische Situation einer ,Sättigung'; das heißt, bei geringer Ungleichheit sind Umverteilungswünsche niedrig. Aber weder der amerikanische, noch der postsozialistische ,median voter ${ }^{6}$ reagiert theorieadäquat. ${ }^{21}$ Beide Male handelt es sich um Länder mit markanten so-

21 Siehe auch den Befund einer Studie, die zeigt, dass objektiv zunehmende Ungleichheit nicht begleitet wird von zunehmenden Umverteilungspräferenzen (Kenworthy/ MacCall 2007). zialpolitischen Ideologien, derentwegen objektive Bedingungen offenbar nicht zum Zuge kommen. Die bivariate Analyse zeigt vorerst, dass empirische Umverteilungspräferenzen von beiden großen Theoriesträngen (Regime oder Politische Ökonomie) einerseits nur teilweise erklärt werden, dass diese andererseits aber durchaus aussagekräftig sind. Die anschließende multivariate Analyse soll das Zusammenspiel beider genauer zeigen.

\section{Individuelle und länderspezifische Determinanten der Zustimmung zu Umverteilung}

Mit einer multivariaten Multilevel-Regression auf der Grundlage aller 23 Länder wird nun geschätzt, auf welche Faktoren die zwischen den Bürgern verschiedener Länder variierende Toleranz staatlicher Umverteilung zurückzuführen ist. Welchen Einfluss haben die makro-ökonomischen Rahmenbedingungen, die Regimezugehörigkeit und die individuellen Merkmale auf die Zustimmung zu Umverteilung? Tabelle 1 zeigt die Ergebnisse mehrerer Schätzungen. Das Null-Modell (Random Intercept Only = RIO) enthält noch keinerlei erklärende Variablen und ist ein ANOVA-Modell. Der durchschnittliche Wert des Indexes zur Akzeptanz von Umverteilung beträgt 7,98. Das RIO-Modell zeigt, dass die Varianz der länderspezifischen Konstanten signifikant ist. Auf der Basis des RIO-Modells lässt sich der Intraklassenkoeffizient (IKK), der über den Anteil der länderspezifischen Varianz an der Gesamtvarianz informiert, berechnen. ${ }^{22}$ Danach lässt sich $17 \%$ der

${ }^{22}$ Der Anteil der länderspezifischen Varianz an der Gesamtvarianz wurde mit dem Intraklassen-Korrelationskoeffizienten (IKK) nach dem Verfahren von Snijders/Bosker (1999), wie bei Hans (2006) beschrieben, berechnet. Der Koeffizient beträgt 0,170 . 
Tabelle 1 Multilevel Regression für Zustimmung zu Umverteilung (Regressionskoeff.)

\begin{tabular}{|c|c|c|c|c|c|}
\hline & RIO-Modell & BIP, Gini & Regime & $\begin{array}{l}\text { Regime, } \\
\text { BIP, Gini }\end{array}$ & $\begin{array}{l}\text { RS-Modell } \\
\text { Kontext- + } \\
\text { indiv. Var. }\end{array}$ \\
\hline Konstante & $\begin{array}{l}7.98 * * * \\
(.164)\end{array}$ & $\begin{array}{l}9.87^{* * *} \\
(.648)\end{array}$ & $\begin{array}{l}7.34 * * * \\
(.227)\end{array}$ & $\begin{array}{l}8.60 * * * \\
(.797)\end{array}$ & $\begin{array}{l}9.65 * * * \\
(.707)\end{array}$ \\
\hline \multicolumn{6}{|l|}{ Fixe Parameter } \\
\hline Gini & & $\begin{array}{l}-1.242 \\
(1.943)\end{array}$ & & $\begin{array}{c}-.448 \\
(1.838)\end{array}$ & $\begin{array}{l}-1.351 \\
(1.604)\end{array}$ \\
\hline BIP (pro Kopf, PPP) & & $\begin{array}{l}-.082^{* * *} \\
(.013)\end{array}$ & & $\begin{array}{c}-.045^{*} \\
(.020)\end{array}$ & $\begin{array}{r}-.034^{*} \\
(.014)\end{array}$ \\
\hline $\begin{array}{l}\text { Regime, Dummy, Ref. sozdem. } \\
\text { - Liberal }\end{array}$ & & & $\begin{array}{l}-.222 \\
(.271)\end{array}$ & $\begin{array}{l}-.216 \\
(.255)\end{array}$ & $\begin{array}{l}.108 \\
(.223)\end{array}$ \\
\hline - Konservativ & & & $\begin{array}{l}.443 \\
(.338)\end{array}$ & $\begin{array}{l}.387 \\
(.307)\end{array}$ & $\begin{array}{l}.683^{*} \\
(.269)\end{array}$ \\
\hline - Mediterran & & & $\begin{array}{l}.957^{* *} \\
(.303)\end{array}$ & $\begin{array}{l}.649 * \\
(.319)\end{array}$ & $\begin{array}{l}.535^{*} \\
(.280)\end{array}$ \\
\hline - Post-sozialistisch & & & $\begin{array}{l}1.31 * * * \\
(.268)\end{array}$ & $\begin{array}{l}.689 * \\
(.370)\end{array}$ & $\begin{array}{l}.697^{*} \\
(.324)\end{array}$ \\
\hline - Haushaltseinkommen (1-10) & & & & & $\begin{array}{l}-.081 * * * \\
(.012)\end{array}$ \\
\hline - Bildung in Jahren & & & & & $\begin{array}{l}-.053 * * * \\
(.003)\end{array}$ \\
\hline $\begin{array}{l}\text { - Soziale Position } \\
\qquad(1=\text { hoch, } 10=\text { niedrig })\end{array}$ & & & & & $\begin{array}{l}.137^{* * *} \\
(.007)\end{array}$ \\
\hline - Geschlecht (Ref. weiblich) & & & & & $\begin{array}{l}-.196 * * * \\
(.039)\end{array}$ \\
\hline - Ungleichheit + Wohlstand & & & & & $\begin{array}{l}-.304 * * * \\
(.010)\end{array}$ \\
\hline - Gini * Hausheinkommen & & & & & $\begin{array}{l}.001 \\
(.002)\end{array}$ \\
\hline - BIP * Hausheinkommen & & & & & $\begin{array}{l}-.005^{* * *} \\
(.001)\end{array}$ \\
\hline $\begin{array}{l}\text { Varianzkomponenten } \\
\text { Ebene } 1 \text { Residualvarianz }\end{array}$ & 1.205 & $2.869(.025)$ & $2.869(.252)$ & $1.205(.0)$ & $2.526(.024)$ \\
\hline $\begin{array}{l}\text { Ebene } 2 \text { Konstante } \\
\text { Hausheinkommen } \\
\text { Geschlecht } \\
\text { Cov (Hausheink.-Geschlecht) } \\
\text { Cov (Geschlecht-Konstante) } \\
\text { Cov (Hausheink.-Konstante) }\end{array}$ & $.1796()$. & $.213(.063)$ & $.184(.055))$ & $.036(.0)$ & $\begin{array}{r}.185(.084) \\
.003(.000) \\
.024(.010) \\
.005(.003) \\
-.011(.008) \\
-.042(.026)\end{array}$ \\
\hline LL Log-Likelihood & -50691.82 & -50680.23 & -50678.58 & -50676.32 & -42786.62 \\
\hline 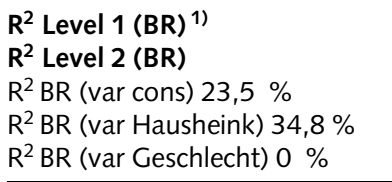 & IKK $17 \%$ & $63,7 \%$ & $68,7 \%$ & $74,3 \%$ & $\begin{array}{l}11,9 \% \\
78,8 \%(S B)\end{array}$ \\
\hline $\mathrm{N}$ & 26017 & 26017 & 26017 & 26017 & 22692 \\
\hline
\end{tabular}

Quelle: ISSP 1999;HDR, LIS; eigene Berechnung, ${ }^{*} \mathrm{p}<0,05 ;{ }^{*} \mathrm{p} 0.01 ;{ }^{*} * \mathrm{p}$ 0.001; Standardfehler in Klammern.

1) $R^{2}$ bzw. Anteil erklärter Varianz wurde berechnet nach Bryk/Raudenbush; lediglich $R^{2}$ derLevel2 Variablen im RS-Modell wurde nach Snider/Bosker berechnet; siehe Hans (2006). 
Gesamtvarianz auf die Länderebene zurückführen. Individuelle Merkmale tragen demnach deutlich mehr als die der Länderebene zur Gesamtvarianz der Umverteilungspräferenzen bei. Im Folgenden wird analysiert, wie diese $17 \%$ der Gesamtvarianz durch die verschiedenen Indikatoren für die länderspezifischen Einflüsse zu erklären sind.

Im zweiten Modell werden nur die nach den Ansätzen der Politischen Ökonomie wichtigen Variablen (Gini, BIP pro Kopf) eingeführt. Der Gini-Index wird nicht signifikant, auch bei Kontrolle der Regime im dritten Modell nicht. Nach dem höchst signifikanten und negativen Koeffizienten für das BIP sind es - vorbehaltlich des noch zu kontrollierenden Einflusses der Regime und der Individualvariablen - die Wirtschaftskraft und der relative Wohlstand, der Umverteilungswünsche der Bürger steigen oder fallen lässt. Mit steigender wirtschaftlicher Entwicklung nimmt die Unterstützung von Umverteilung ab, was Hypothese drei, wonach mit steigender Wohlfahrt soziale Probleme als weniger gravierend wahrgenommen werden und daher die Forderung von Umverteilung abnimmt (Sättigung), bestätigt.

Das Modell, das nur die Zugehörigkeit der Befragten zu den Regimen enthält (Dummy-Variable, Kontrast sozialdemokratische Regimes), weist für post-sozialistische und in geringerem Maße auch für mediterrane Regime signifikant höhere Umverteilungspräferenzen im Vergleich zu den skandinavischen Regimen aus. Die Regressionskoeffizienten für die liberalen und die konservativen Regime sind hingegen nicht signifikant. Demnach differiert nur das Niveau der Umverteilungswünsche in unreifen Wohlfahrtsstaaten und Ländern mit niedrigem BIP, die Nachzügler der wohlfahrtsstaatlichen Entwicklung sind (Gelissen 2002), deutlich von dem Niveau in sozialdemokratischen Ländern. Wechseln wir deshalb gleich zum nächsten Modell, in dem der Einfluss der Regime durch die beiden ökonomischen Faktoren BIP und Gini kontrolliert wird. Dadurch wird der höchst signifikante Effekt der postsozialistischen Länder geringer, verschwindet aber nicht. Das heißt, dass zusätzlich die geringe Wirtschaftskraft und nicht allein sozialistische Sozialpolitikideen die Umverteilungsforderungen der Bürger nährt. Gleichzeitig verliert auch das BIP an Erklärungskraft, ohne dass diese ganz verschwindet. Einerseits bleibt bei Kontrolle der ökonomischen Aspekte nur ein kleiner regimespezifischer Einfluss übrig, andererseits erweisen sich Umverteilungswünsche weniger gut durch ökonomische Merkmale erklärbar, wenn zusätzlich die Regimezugehörigkeit der Befragten berücksichtigt wird.
Die von der ersten Hypothese angenommenen regimespezifischen Umverteilungspräferenzen und eine Rangordnung mit sozialdemokratischen Regimes an zweiter Stelle ist somit nur partiell bestätigt. Diese Hypothese ist offenbar zu einfach, da die den formativen Effekt der Regime überlagernden Faktoren nicht berücksichtigt wurden. Allerdings darf man aus dem Fehlen eines signifikanten Effektes der liberalen Regime keineswegs folgern, dass Ungleichheit und Umverteilung von Bürgern liberaler und sozial-demokratischer Regime gleich beurteilt würden. Vielmehr geht der fehlende signifikante Effekt teils auf die Inkohärenz der Unterstützung für Umverteilung innerhalb des liberalen Regimes selbst zurück. Da Umverteilung in Großbritannien sehr viel akzeptierter ist als in den USA (siehe Tab. A1 Anhang), verwischen bei der Zusammenfassung zu Regimes die durchaus bestehenden internen Unterschiede. ${ }^{23}$ Teils ist jedoch das Unterstützungsniveau für Umverteilung in sozialdemokratischen Ländern tatsächlich eher gering, was selbstverständlich von der bereits in hohem Maße institutionalisierten Umverteilung verursacht wird, nicht von der Akzeptanz der ungleichen Einkommensverteilung.

Vergleicht man den durch die Kontextvariablen erklärten Anteil der Varianz der Umverteilungspräferenzen, dann sieht man, dass das zweite Modell mit den für die Politische Ökonomie wichtigen Variablen sogar etwas weniger Varianz erklärt als das Modell mit den Regime-Variablen. Beide erfassen jeweils aber bereits zwei Drittel der Varianz auf Länderebene. Zusammengenommen können ökonomische Merkmale und die Information über das Regime sogar ca. 74 \% der zwischen Ländern variierenden Umverteilungspräferenzen erklären. Man verfügt mit beiden Variablentypen also bereits über ein recht gutes Modell zur Erklärung der unterschiedlichen Umverteilungswünsche.

Im letzten RS-Modell kommen erklärende Variablen der individuellen Ebene (Level 1), die Interaktionseffekte und die Random Slopes für Einkommen und Geschlecht zu den Kontextvariablen dazu. Dies steigert die erklärte Varianz der zwischen den Ländern schwankenden Umverteilungspräferenzen nochmals leicht auf etwa $79 \%$. Durch das Berücksichtigen von Cross-Level-Effekten für das Einkom-

\footnotetext{
${ }^{23}$ Die geringe sozialstaatliche Umverteilung und die auch geringen Forderungen nach Umverteilung kennzeichnen die USA. Die Forschung entwickelte spezifische, auf Besonderheiten des Landes eingehende Erklärungen, die über die Annahme einer liberalen Ideologie hinausgehen (Alesina et.al. 2001).
} 
men im Kontext verschiedener wirtschaftlicher Wohlfahrt und durch die Möglichkeit eines zwischen den Ländern variierenden Effektes des Einkommens im RS-Modell ergeben sich vor allem zwei Veränderungen: Erstens erhalten selbst konservative Regime einen signifikanten Koeffizienten, d. h. sogar hier ist der Redistributionswunsch (nach den bivariaten Grafiken v. a. wegen Frankreich und Österreich) im Vergleich zu sozialdemokratischen Ländern höher. Zweitens bringt das RS-Modell eine im Vergleich zum RI-Modell mit allen Kontextvariablen um $23,5 \%$ verbesserte Varianzaufklärung. Je größer das BIP ist, desto stärker negativ wirkt sich demnach ein steigendes Haushaltseinkommen auf die Umverteilungspräferenzen aus.

Die faktische Ungleichheit hat auch nach dem letzten Modell keinen signifikanten Einfluss auf die Bewertung von Ungleichheit und Umverteilungswünsche. Die zweite Hypothese in Anschluss an das Richard-Melzer-Theorem, wonach die Unterstützung für Umverteilung um so größer ist, je höher die Ungleichheit in einem Land ausfällt und je mehr median voters daher ein Interesse an Umverteilung haben, ist damit so generell nicht bestätigt. Die Zustimmung zu Umverteilung resultiert nicht direkt aus der objektiven Ungleichheit, sondern vermittelnde Faktoren sind gesucht, wie eben kulturell geprägte Bewertungen von Ungleichheit und der Verantwortung des Staates bei der Angleichung der Einkommen (Lübker 2007). Um den nicht signifikanten Koeffizienten für den Gini-Index angemessen zu interpretieren, muss noch einmal auf das Ergebnis von Abbildung 2 verwiesen werden. Dort wurde deutlich, dass die gegebene Ungleichheit in manchen Ländern durchaus Umverteilungspräferenzen beeinflusst, aber eben nicht in allen. Da dies aber nicht in allen Ländern der Fall ist, ergibt sich bei der multivariaten Analyse kein signifikanter Koeffizient. Die disparaten Tendenzen in liberalen und postsozialistischen Ländern heben diese Beziehung zwischen niedrigem Gini und ebenso niedriger Unterstützung von Umverteilung auf.

Betrachtet man weiter die Koeffizienten der Individualvariablen, dann sieht man, dass die meisten unabhängigen Variablen, die eine besondere $\mathrm{Ab}$ hängigkeit der Personen von staatlicher Einkommensumverteilung indizieren, signifikant sind und das theoretisch erwartete Vorzeichen haben. Je geringer das Familieneinkommen ausfällt, je geringer das Niveau der schulischen Bildung ist und je geringer die eigene soziale Position eingeschätzt wird, desto höher sind die Umverteilungspräferenzen. Interpretiert man das Niveau schulischer Bildung auch als Indikator für Arbeitsmarktchancen, dann steigt bei schlechten Arbeitsmarktchancen die Unterstützung von Umverteilung. Die vierte Hypothese, wonach Umverteilungspräferenzen von den individuellen Vor- und Nachteilserwartungen beeinflusst werden, ist dennoch im großen und Ganzen bestätigt. Deutlich ist wegen des hoch signifikanten Regressionskoeffizienten auch der Einfluss des Geschlechts: Männer unterstützen weniger als Frauen die sozialstaatliche Redistribution von Einkommen. Neben den Interessen haben Ideen über Ungleichheit einen deutlichen Einfluss auf Umverteilungspräferenzen. Wie erwartet, lässt eine positive Sicht von Ungleichheit (sie fördere wirtschaftliche Wohlfahrt) die Zustimmung zu Umverteilung sinken. Der mit der fünften Hypothese angenommene Einfluss ideeller Orientierungen ist somit bestätigt. Alle individuellen Variablen erklären $11,9 \%$ der Varianz der Unterstützung von Umverteilung.

Schließlich bleibt noch auszuwerten, ob es ein Mehr an Erklärungskraft bringt, einige unabhängige Variablen in den zufälligen Teil der Multilevel-Regression aufzunehmen. Nach unseren Annahmen sollten die Steigungen der Variablen Familieneinkommen und Geschlecht zwischen den Ländern schwanken. Mit dem Devianz-Test (oder Likelihood-Ratio-Test) wurde geprüft, ob sich ein vollständiges Modell mit allen erklärenden Variablen und Zufallskomponente und ein vollständiges Modell ohne Zufallskomponente überhaupt unterscheiden. Das ist in der Tat der Fall, da die Differenz zwischen beiden hoch signifikant ist. ${ }^{24}$ Das Haushaltseinkommen erklärt 34,8 \% der Varianz der Regressionskonstante, das Geschlecht bewirkt keine unterschiedlichen Effekte; demnach formiert sich auch keine Cleavage-Gruppe der Frauen in den sozialdemokratischen Regimen, die besonders stark Umverteilung unterstützen.

Insgesamt kommt man zu ambivalenten Resultaten bezüglich der Erklärung der in diesem Aufsatz im Zentrum stehenden Erklärung von Länderunterschieden. Die hohe wirtschaftliche Leistung des Landes lässt zwar den Umverteilungsbedarf schrumpfen, determiniert jedoch nichts. Die Spielräume, die unter Bürgern ,reicher' Länder auftreten, unterstrei-

\footnotetext{
${ }^{24}$ Diese länderspezifische Varianz der Steigung läßt sich eingehender prüfen: Die Koeffizient des Familieneinkommens schwanken zwischen -0.187 und 0,057. Eine Kovarianz zwischen dem Einkommen und dem Geschlecht gibt es hingegen kaum; ebenfalls gibt es keinen Hinweis auf eine Kovarianz zwischen dem Familieneinkommen und der Varianz der Umverteilungspräferenzen (d. h. der Konstante).
} 
chen die Rolle nicht-ökonomischer, ideeller Gründe für die Unterstützung staatlicher Einkommensumverteilung. Ebenso wenig können allein Regime als Ursache unterschiedlicher Akzeptanz von Ungleichheit und Umverteilung postuliert werden. Dies würde zu kurz greifen und der Bevölkerung des weitreichend umverteilenden Schweden etwa keine rationale Anpassung ihrer durchaus hohen Umverteilungspräferenzen zugestehen. Es gibt keine uniforme Tendenz in den Daten, die die eine oder die andere theoretische Erklärung bestätigt. Diese Ambivalenz und die Tatsache, dass die Regressionskoeffizienten der Ländermerkmale das Signifikanzniveau nur knapp unterschritten, schmälern aber nicht den Wert der vorgelegten Analyse. Diese zeigt vielmehr eine partielle, aber eingeschränkte Gültigkeit der beiden theoretischen Stränge: Keiner allein bietet eine angemessene Erklärung der Muster in den empirischen Daten. In Kombination zeigen sie jedoch ein hohes Maß an Erklärungskraft, denn die Variablen der zweiten Ebene erklären $79 \%$ der Varianz der Daten, die überhaupt durch Länder verursacht wird.

\section{Diskussion und Schlussfolgerungen}

Dieser Beitrag ging davon aus, dass die von der Forschung oft bestätigten Länderunterschiede in den Umverteilungspräferenzen der Bürger durch den in der politischen Soziologie dominierenden Regimeansatz nicht ausreichend erklärt werden, da diese Unterschiede ohne Kontrolle weiterer Erklärungen und entsprechender empirischer Faktoren alleine dem Einfluss des Wohlfahrtsregimes zugeschrieben werden. Die variierende Akzeptanz von Umverteilung, die man auf der Ebene der Länder findet, wurde aber mit dem Regimeeinfluss nicht plausibel. Der Beitrag erweiterte die theoretische Perspektive um Ansätze aus der Politischen Ökonomie, die verschiedene Umverteilungswünsche der Bürger als Reaktionen auf das Niveau an Ungleichheit oder den wirtschaftlichen Wohlstand in verschiedenen Ländern zustande kommen sehen. Aber auch diese Ansätze stoßen an Grenzen bei der Erklärung der empirischen Muster der Umverteilungspräferenzen, die offenbar nicht unmittelbar auf objektive Faktoren reagieren. Eine solche unvermittelte Beziehung unterstellt aber die median voter-Hypothese mit der Annahme, dass mit steigender Ungleichheit ebenfalls das Niveau der Forderung nach Umverteilung in der Bevölkerung wachse, da mehr Menschen individuelle Vorteile davon erwarten. Die Unterstützung für Umverteilung in den liberalen
Ländern USA, Kanada oder Australien entspricht aber nicht dieser Hypothese. Dieser Befund wurde in der Sozialforschung vielfach aufgegriffen, teils als Beleg für die zentrale Rolle von Ideen und Ideologien, teils auch innerhalb der politischen Ökonomie, wo alternative Erklärungsansätze entwickelt wurden. Die starken ethnischen Spaltungen wurden als Grund der geringen Umverteilungsforderungen der Amerikaner gesehen, was an sich hoch plausibel ist. Die diesem Beitrag zugrunde liegende Datenanalyse bestätigte den Einfluss der ethnischen Fraktionalisierung jedoch nicht, da dieser wegen der großen Zahl an einbezogenen Ländern mit heterogenen ethnischen Strukturen nicht zutage treten konnte.

Auch der vom Grad der Ungleichheit erwartete Effekt verschwimmt und zeigt sich empirisch nur undeutlich wegen der in verschiedenen Ländern variierenden Wirkung. Wie nach dem median voterModell angenommen, findet man bei Ländern mit einem niedrigen Gini-Koeffizienten auch eine geringere Unterstützung für weitere Umverteilung. Umgekehrt entwickeln sich jedoch bei hoher Ungleichheit nicht automatisch Forderungen nach umverteilender Sozialpolitik, obwohl doch in der Einkommenshierarchie schlechter gestellte Wähler individuelle Vorteile erwarten könnten. Dieser für die liberalen angelsächsischen Länder geltende Befund verweist auf den Einfluss sozialpolitischer Ideologien und Wertvorstellungen, die jedenfalls in dieser Ländergruppe stärker sind als Vorteilserwartungen. Auch in post-sozialistischen Ländern scheint die Ideologie über die objektiven Anreize zu dominieren, da hier trotz relativ hoher Gleichheit in der Bevölkerung dennoch das im Vergleich zu anderen Ländern höchste Niveau der Umverteilungsforderungen zu finden war. Folglich ist die sozialpolitische Ideenwelt in den verschiedenen Varianten des Wohlfahrtskapitalismus durchaus einflussreich. Bestimmte Wahrnehmungskategorien für Gerechtigkeit oder Gleichheit schalten sich vor die objektive Ungleichheit. Ohne solche vermittelnde, regimespezifischen Kategorien lassen sich sozialpolitische Präferenzen folglich nicht begreifen. Aber generalisieren sollte man das Konzept der politischen Soziologie nicht, da der erwartete Rückgang der Umverteilungswünsche in den Ländern mit egalitäreren Einkommensstrukturen durchaus eintritt. Bürger passen teils ihre Umverteilungsforderungen den ökonomischen Gegebenheiten an. Im Vergleich zum Einfluss des Gini-Koeffizienten war der Einfluss des Wohlstandsniveaus auf die Umverteilungspräferenzen recht eindeutig: Ein hoher wirtschaftlicher Wohlstand pro Kopf führt in der Tat dazu, 
dass Ungleichheit als weniger gravierend eingestuft wird und die Forderung nach Umverteilung in der Bevölkerung sinkt. Das Resultat gilt auch bei Kontrolle des Gini-Koeffizienten, weshalb allein schon ein hohes Bruttosozialprodukt pro Kopf auch ohne Berücksichtigung der Einkommensverteilung die Redistributionsforderungen mindert, da mit Ungleichheit zusammenhängende Probleme offenbar weniger scharf wahrgenommen werden. Eine starke Wirtschaft könnte aber auch die Überzeugung wachsen lassen, dass jeder im Grunde die Chance hat, etwas vom Wohlstand abzubekommen.

Nach dem Regimeansatz muss es eine gewisse Kohärenz zwischen den in sozialstaatlichen Programmen institutionalisierten Leitideen zu Ungleichheit einerseits und den Umverteilungsforderungen der Bürger andererseits geben. Teilweise spiegeln zwar die Ergebnisse meiner Analyse eine solche Kohärenz, aber diese ist keineswegs generell gegeben. Der Einfluss der sozialpolitischen Regime auf die Haltung der Bürger zu Ungleichheit und Umverteilung wird im Regimekonzept zu eng gedacht. Auch sieht die Rangreihe der öffentlichen Zustimmung zu Umverteilung nicht so aus, wie man nach dem Regimeansatz erwarten würde.
Insgesamt kommt man zu dem Resultat, dass sich der Regimeansatz der politischen Soziologie und die Erklärungsansätze der politischen Ökonomie eher ergänzen als ausschließen. Keine der beiden Theorien allein erfasst adäquat die Länderunterschiede in den Einschätzungen von Ungleichheit und Umverteilungsforderungen, die die vergleichende Forschung zu public opinions schon lange beschreibt. Die durch ein kulturelles Erbe geprägten Akteure lassen sich nicht gegen rationale Akteure ausspielen. Künftige Forschung müsste den Einfluss weiterer theoretisch sinnvoller Ländermerkmale testen.

Letztlich darf man nicht vergessen, dass der größte Teil der Varianz in den Umverteilungspräferenzen auf individuelle Merkmale zurückgeht, und nur der kleinere auf Niveauunterschiede zwischen Ländern. Mit den in der Forschung bislang geprüften individuellen Faktoren wie dem Einkommen oder der sozialen Position ließ sich aber nur ein geringer Teil der Varianz der Daten erklären. Die weitere Forschung muss auch auf der individuellen Ebene ansetzen und bessere Erklärungsmodelle entwickeln.

\section{Anhang}

Tabelle A1 Mittelwerte des Index zu Umverteilungsbereitschaft und der einzelnen Variablen des Index (Rangordnung)

\begin{tabular}{|c|c|c|c|c|c|}
\hline Land & $\begin{array}{c}\text { Index, } \\
\text { abhängige Var. }\end{array}$ & $\begin{array}{l}\text { Standard- } \\
\text { abweichung }\end{array}$ & $\begin{array}{l}\text { Ungleichheit } \\
\text { zu hoch }\end{array}$ & $\begin{array}{l}\text { Staat soll } \\
\text { Ungleichheit } \\
\text { reduzieren }\end{array}$ & $\mathbf{N}$ \\
\hline Portugal & 9,27 & 1,253 & 4,8 & 4,5 & 1123 \\
\hline Russland & 9,12 & 1,362 & 4,8 & 4,4 & 1613 \\
\hline Bulgarien & 9,11 & 1,367 & 4,8 & 4,3 & 1048 \\
\hline Ungarn & 8,77 & 1,411 & 4,6 & 4,2 & 1180 \\
\hline Slovakei & 8,68 & 1,439 & 4,7 & 4,0 & 1123 \\
\hline Slovenien & 8,55 & 1,422 & 4,4 & 4,2 & 975 \\
\hline Lettland & 8.51 & 1,318 & 4,5 & 4,8 & 1050 \\
\hline Polen & 8,47 & 1,464 & 4,3 & 4,2 & 1045 \\
\hline Czech. Rep. & 8,33 & 1,865 & 4,4 & 3,9 & 1766 \\
\hline Deutschland-Ost & 8,29 & 1,410 & 4,4 & 3,9 & 487 \\
\hline Spanien & 8,23 & 1,431 & 4,2 & 4,0 & 1172 \\
\hline Frankreich & 8,11 & 1,861 & 4,4 & 3,7 & 1844 \\
\hline Österreich & 8,10 & 1,663 & 4,3 & 3,9 & 951 \\
\hline Großbritannien & 7,74 & 1,621 & 4,1 & 3,7 & 752 \\
\hline Schweden & 7,46 & 1,968 & 3,9 & 3,6 & 1105 \\
\hline Japan & 7,36 & 2,180 & 3,9 & 3,5 & 1174 \\
\hline
\end{tabular}


Tabelle A1 (Fortsetzung)

\begin{tabular}{|c|c|c|c|c|c|}
\hline Land & $\begin{array}{c}\text { Index, } \\
\text { abhängige Var. }\end{array}$ & $\begin{array}{l}\text { Standard- } \\
\text { abweichung }\end{array}$ & $\begin{array}{l}\text { Ungleichheit } \\
\text { zu hoch }\end{array}$ & $\begin{array}{l}\text { Staat soll } \\
\text { Ungleichheit } \\
\text { reduzieren }\end{array}$ & $\mathrm{N}$ \\
\hline Norwegen & 7,35 & 1,905 & 3,8 & 3,6 & 1213 \\
\hline Neuseeland & 7,12 & 2,061 & 3,8 & 3,2 & 1038 \\
\hline Zypern & 7,16 & 1,613 & 3,7 & 3,5 & 966 \\
\hline Deutschland-West & 7,13 & 1,834 & 3,8 & 3,3 & 813 \\
\hline Australien & 7,03 & 1,758 & 3,8 & 3,3 & 1583 \\
\hline Kanada & 6,86 & 2,057 & 3,8 & 3,1 & 919 \\
\hline USA & 6,69 & 1,909 & 3,8 & 2,9 & 1159 \\
\hline Total & 8,00 & 1,862 & 4,2 & 3,8 & 26017 \\
\hline
\end{tabular}

Quelle: ISSP 1999, eigene Berechnung

Tabelle A2 Deskriptive Statistik der in der Analyse verwendeten Variablen

\begin{tabular}{lcc}
\hline Abhängige Variablen & arith. Mittel & Standardabw. \\
Einkommensunterschiede sind zu groß & 4,21 & 1,18 \\
\hline Staat soll Einkommensunterschiede reduzieren & 3,78 & 1,14 \\
Unabhängige Variablen & \\
Ungleichheit fördert Wohlstand aller & 2,39 \\
Geschlecht männlich in \% (Ref. weiblich) & 47,9 \\
Bildung in Jahren & $12,3(4,31)$ \\
Soziale Position (1 = hoch, 10= niedrig) & $6,0(1,88)$ \\
Haushaltseinkommen (in Dezilen 1-10) & $5,5(2,38)$ \\
Sozialausgaben in \% des BIP & $20,3(4,93)$ \\
BIP pro Kopf 1999 (in Dollar, PPP) & $18,34(7,54)$ \\
Gini & $0,317(5,81)$ \\
Regime (Anteil der Befragten im jeweiligen Regime an den Befragten in \%) & \\
- Sozialdemokratisch & 9,3 \\
- Konservativ & 13,9 \\
- Liberal & 25,7 \\
- Postkommunistisch & 38,9 \\
\hline
\end{tabular}

\section{Literatur}

Alesina, A. / Glaeser, E. / Sacerdote, B., 2001: Why Doesn't the United States Have A European-Style Welfare State? Brookings Papers on Economic Activity 2: 187-254.

Andress, H.J. / Heien, T., 2001: Four Worlds of Welfare State Attitudes? A Comparison of Germany, Norway, and the United States. European Sociological Review 17: 337-356.

Atkinson, T. / Rainwater, L. / Smeeding, T.M., 1995: Income Distribution in OECD Countries. Evidence from the Luxembourg Income Study. Social Policy Studies 18, OECD Paris.

Arts, W. / Gelissen, J., 2001: Welfare States, Solidarity and
Justice Principles: Does the Type really matter? Acta Sociologica 44: 283-299.

Bayertz, K., 1998: Begriff und Problem der Solidarität. S. 11-53 in: ders. (Hrsg.), Solidarität: Begriff und Problem. Frankfurt a.M.: Suhrkamp.

Becker, U. / Kersbergen, K. von, 1986: Der christliche Wohlfahrtsstaat der Niederlande - ein kritischer Beitrag zur vergleichenden Politikforschung. Politische Vierteljahresschrift 27: 61-77.

Birkel, C., 2005: Einkommensungleichheit und Umverteilung in Westdeutschland, Großbritannien und Schweden, 1950-2000. Luxembourg Income Study, Working Paper No. 425. (lisproject.org)

Blekesaune, M. / Quadagno, J., 2003: Public Attitudes Toward Welfare State Policies. A Comparative Analysis 
of 24 Nations. European Sociological Review 19: 415-427.

Bowles, S. / Gintis, H., 2000: Reciprocity, self-interest, and the welfare state. Nordic Journal of Political Economy 26: 33-53.

Braun, M., 1998: Soziale Ungleichheit und Wohlfahrtsstaat. Einstellungswandel in Ost- und Westdeutschland. S. 115-138 in: M. Braun / P. Mohler (Hrsg.), Blickpunkt Gesellschaft 4. Soziale Ungleichheit in Deutschland. Opladen. Leske+Budrich.

Brooks, C. / Manza J., 2006: Social Policy Responsiveness in Developed Democracies. American Sociological Review 71: 474-494.

Corneo, G. / Gruner, H.-P., 2002: Individual Preferences for Political Redistribution. Journal of Public Economics 83: 83-107.

Delhey, J., 1998: Inequality and Support for redistributive Policy: One World of Post-Communism, Two Worlds of Western Capitalism. Rep./Working Paper FS III 98-401. Wissenschaftszentrum Berlin für Sozialforschung.

Engel, U., 1998: Einführung in die Mehrebenenanalyse. Grundlagen, Auswertungsverfahren und praktische Beispiele. Opladen.

Esping-Andersen, G., 1996 [1990]: The three worlds of welfare capitalism. Cambridge: Polity Press.

Finseraas, H., 2006: Income Inequality and Demand for Redistribution. An Empirical Analysis of European Public Opinion. Paper presented at the ESPAnet conference Bremen 2006.

Förster, M. / d'Ercole, M.M., 2005: Income Distribution and Poverty in OECD Countries in the Second Half of the 1990s. OECD social, employment and migration working papers No. 22. (www.oecd.org).

Gelissen, J. (2002): Worlds of Welfare, Worlds of Consent? Public Opinion on the Welfare fare State. London: Allan and Unwin.

Hall, P.A. / Soskice, D. (Hrsg.), 2001: Varieties of Capitalism. The Institutional Foundations of Comparative Advantage. Oxford. University Press.

Haller, M. / Mach, B. / Zwicky, H., 1995: Egalitarismus und Anti-egalitarismus zwischen gesellschaftlichen Interessen und kulturellen Leitbildern. Ergebnisse eines internationalen Vergleichs. S. 221-264 in: H.P. Müller / B. Wegener (Hrsg.), Soziale Ungleichheit und soziale Gerechtigkeit. Opladen: Leske+Budrich.

Hans, S., 2006: Die Analyse gepoolter Daten mit Mehrebenenmodellen - Einstellungen zu Zuwanderern im europäischen Vergleich. Berliner Studien zur Soziologie Europas. BSSE-Arbeitspapier Nr. 6, Freie Universität Berlin.

Hinrichs, K., 1997: Social Insurances and the Culture of Solidarity. The Moral Infrastructure of Interpersonal Redistribution. ZeS Arbeitspapier Nr. 3/97. Universität Bremen.

Human Development Report, 2003: (http://siteresources. worldbank.org/INTWDR2003/Ressources/complete report.pdf)

Iversen, T. / Soskice, D., 2001: An Asset Theory of Social Policy Preferences. American Political Sciences Review 95: 875-893.
Iversen, T. / Soskice, D., 2006: Electoral Institutions and the Politics of Coalitions. Why Some Democracies Redistribute More Than Others. American Political Sciences Review 100: 165-181.

Kangas, O. E., 2003: The grasshopper and the ants: popular opinions of just distribution in Australia and Finland. The Journal of Socio-Economics 31: 721-743.

Kaufmann, F.X., 1997: Herausforderungen des Sozialstaats. Frankfurt a.M.: Suhrkamp.

Kaufmann, F.-X., 2003: Varianten des Wohlfahrtsstaates. Der deutsche Sozialstaat im internationalen Vergleich. Frankfurt a.M.: Suhrkamp.

Kenworthy, L. / Pontusson, J., 2005: Rising Inequality and the Politics of Redistribution in Affluent Countries. Perspectives on Politics 3: 449-471.

Kenworthy, L. / McCall, L., 2007: Inequality, Public Opinion, and Redistribution. Paper http://www.u.arizona.edu/lkenwor/. Forthcoming in Socio Economic Review.

Kluegel, J. / Miyano, M., 1995: Justice Beliefs and Support for the Welfare State in Advanced Capitalism. S. 81-105 in: J. Kluegel / D.S. Mason / B. Wegener (Hrsg.), Social Justice and Political Change. Berlin, New York: de Gruyter.

Korpi, W., 1983: The democratic class struggle. London: Routledge.

Korpi, W. / Palme, J., 1998: The Paradox of redistribution and strategies of equality: Welfare State Institutions, Inequality, and poverty in the western countries. American Sociological Review 63: 661-687.

Lessenich, S., 1999: Ein (un)moralisches Angebot: Reziprozitätsfiktionen im modernen Wohlfahrtsstaat. S. 153-168 in: C. Honegger / S. Hradil / F. Traxler (Hrsg.), Grenzenlose Gesellschaft? Opladen: Leske + Budrich.

Lessenich, S. / Ostner, I. (Hrsg.), 1998: Welten des Wohlfahrtskapitalismus. Frankfurt/New York: Campus.

Linos, K. / West, M., 2003: Self-interest, Social Beliefs, and Attitudes to Redistribution. Re-adressing the Issue of Cross-National Variation. European Sociological Review 19: 393-409.

Lippl, B., 2003: Sozialer Wandel, wohlfahrtsstaatliche Arrangements und Gerechtigkeits-äußerungen im internationalen Vergleich. Dissertation. Humboldt Universität Berlin.

Lübker, M., 2007: Inequality and the demand for redistribution: are the assumptions of the new growth theory valid? Socio-Economic Review 5: 117-148.

Mau, S., 1997: Ungleichheits- und Gerechtigkeitsorientierungen in modernen Wohlfahrtsstaaten. Ein Vergleich der Länder Schweden, Großbritannien und der Bundesrepublik Deutschland. WZB Paper FS III 97-401.

Mau, S., 2002: Wohlfahrtsregime als Reziprozitätsarrangements. Berliner Journal für Soziologie 12: 345-364.

Mau, S., 2004: Welfare Regimes and the Norms of Social Exchange. Current Sociology 52: 53-74.

Meier Jaeger, M., 2006a: Welfare Regimes and Attitudes towards Redistribution: The Regime Hypothesis Revisited. European Sociological Review 22: 157-170.

Meier Jaeger, M., 2006b: What makes people support 
public responsibility for welfare provision: Self-interest or political ideology? A longitudinal approach. Acta Sociologica 49: 321-338.

Meltzer, A.H. / Richard, S.F., 1981: A Rational Theory of the Size of Government. Journal of Political Economy 89: 914-927.

Metz, K.H., 1998: Solidarität und Geschichte. Institutionen und sozialer Begriff der Solidarität in Westeuropa im 19. Jahrhundert. S. 172-194 in: K. Bayertz (Hrsg.) Solidarität: Begriff und Problem. Frankfurt a. M.: Suhrkamp.

Moene, K.O. / Wallerstein, M., 2001: Inequality, Social Insurance and Redistribution. American Political Science Review 95: 859-874.

OECD, 2004: Social Expenditure Database. (www.oecd. org/els/social/expenditure.)

Offe, C., 1990: Akzeptanz und Legitimität strategischer Optionen in der Sozialpolitik. S. 179-202 in: C. Sachße / T.H. Engelhardt (Hrsg.), Sicherheit und Freiheit. Zur Ethik des Wohlfahrtsstaats. Frankfurt a.M.: Suhrkamp.

Offe, C., 1998: Der deutsche Wohlfahrtsstaat: Prinzipien, Leistungen, Zukunftsaussichten. Berliner Journal für Soziologie 8: 359-380.

Pettersen, P.A., 1995: The Welfare State: The Security Dimension. S. 198-233 in: O. Borre / E. Scarborough (Hrsg.), The Scope of Government. Oxford: University Press.

Rabe-Hesketh, S. / Skrondal, A., 2005: Multilevel and longitudinal Modeling Using Stata. StataCorp.

Rehm, P., 2005: Citizens Support for the Welfare State. Determinants of Preferences of Income Redistribution. Discussion Paper SP II 2005 - 02 Wissenschaftszentrum Berlin.

Roller, E., 1995: The Welfare State: The Equality Dimension. S. 165-197 in: O. Borre / E. Scarbrough (Hrsg.), The Scope of Government. Oxford University Press.
Rothstein, B., 1998: Just Institutions Matter. The Moral and Political Logic of the Universal Welfare State. Cambridge: Cambridge University Press.

Stjerno, S. / Johannessen, A., 2004: Attitudes of solidarity in Western Europe. Oslo University College. HiO.report no. 23.

Streeck, W., 2000: Wohlfahrtsstaat und Markt als moralische Einrichtungen: Ein Kommentar. S. 135-167 in: K.U. Mayer (Hrsg.), Die beste aller Welten? Marktliberalismus versus Wohlfahrtsstaat. Frankfurt a.M.: Campus.

Svallfors, S., 1997: Worlds of Welfare and Attitudes to Redistribution: A Comparison of Eight Western Nations. European Sociological Review 13: 283-304.

Taylor-Gooby, P., 1983: Legitimation Deficit, Public Opinion and the Welfare State. Sociology 17: 165-184.

Taylor-Gooby, P., 1985: Public Opinion, Ideology and Welfare State. London: Routledge \& Kegan.

Taylor-Gooby, P., 2001: Sustaining State Welfare in Hard Times. Who will foot the bill? Journal of European Social Policy 11: 133-147.

Ullrich, C.G., 1996: Solidarität und Sicherheit. Zur sozialen Akzeptanz der Gesetzlichen Krankenversicherung. Zeitschrift für Soziologie 25: 171-189.

Ullrich, C.G., 2000: Solidarität im Sozialversicherungsstaat. Die Akzeptanz des Solidarprinzips in der gesetzlichen Krankenkasse. Frankfurt a.M./New York: Campus.

Van Oorschot, W., 2000: Why pay for welfare? A sociological analysis of reasons for Welfare. The Netherlands Journal of Social Sciences 36: 15-36.

Autorenvorstellung: Ursula Dallinger, geb. 1959 in Neustadt/Wstr. Studium der Soziologie und Politikwissenschaften in Marburg, Göttingen und Kassel. Promotion in Dortmund. Von 1992-1996 wissenschaftliche Mitarbeiterin an der Universität Dortmund und von 2001-2003 an der Universität Jena. 2003-2007 Post doc an der Universität Hamburg. Seit Dez. 2007 Professorin für Soziologie/Sozialpolitik an der Universität Trier.

Forschungsschwerpunkte: Vergleich von Wohlfahrtsstaaten, Umverteilung und Solidarität im Sozialstaat, Institutionentheorien, Generationenverhältnisse, Alterssoziologie.

Wichtigste Publikationen: Die Wiederentdeckung sozialer Regeln - Institutionen bei Durkheim und North in: ÖZfS 32, 2007: 66-93; Gerechtigkeit zwischen den Generationen in der wohlfahrtsstaatlichen Alterssicherung (mit S. Liebig), in: S. Liebig / H. Lengfeld / S. Mau (Hrsg.), Verteilungsprobleme moderner Gesellschaften. Frankfurt/M., New York 2004; zuletzt in dieser Zeitschrift: Der Konflikt zwischen familiärer Pflege und Beruf als handlungstheoretisches Problem, in: ZfS 27, 1998: 94-112. 